\title{
Interaktive Medien - Ende der Massenkommunikation?
}

\section{Josef Wehner}

Graduiertenkolleg „Interdisziplinäre Strategien zum Schutz der Umwelt, RWTH Aachen

Zusammenfassung: Gegenwärtig sorgt die Vision eines interaktiven Mediensystems für weltweites Aufsehen. Durch die Verbindung von Telekommunikation und Datenverarbeitung sind globale Computernetze entstanden, die es heute jedem mit Zugang zu ihnen ermöglichen, Botschaften nicht nur von unbegrenzt vielen Teilnehmern zu empfangen, sonder auch an ebenso viele zu verteilen. Protagonisten dieser neuen Medienwelt prophezeihen, daB in naher $\mathrm{Zu}-$ kunft das „Einbahnstraßen-System“ der Massenkommunikation (Fernsehen, Massenpresse) in ein "mehrspuriges“ Kommunikationssystem verwandelt wird. Diese Erwartung beruht jedoch auf fragwürdigen Annahmen über die Funktion und Wirkungen der „alten“ Medien. „Ein-Weg-Kommunikation“ übernimmt für die Gesellschaft nicht nur integrative Funktionen, sondern setzt gleichzeitig Reflexions- und Individuierungspotentiale frei, die durch Möglichkeiten der Einmischung der Nutzer in die Medienkommunikation verloren gehen würden. Interaktive Medien werden deshalb Massenkommunikation nicht ersetzen können, jedoch neuartige Kommunikationsmöglichkeiten bieten und das Mediensystem weiter differenzieren.

Es ist nur wenige Jahre her, daß die Vision einer computertechnischen Reproduktion menschlicher Intelligenz heftigste Debatten auslöste. Obwohl immer noch in verschiedenen Teildisziplinen der Kognitionswissenschaften am Projekt „Künstlicher Intelligenz" festgehalten wird, hat dieser Begriff aufgrund des Scheiterns zentraler Leitannahmen das Ende seiner Karriere als Anziehungspunkt öffentlicher Aufmerksamkeit erreicht. Heute ist es die Vorstellung einer Interaktion zwischen nichtanwesenden Personen, die für weltweites Aufsehen sorgt. War es vorher das Denken eines einzelnen Menschen, so sind es nun die kommunikativen Beziehungen zwischen den Menschen, die im Mittelpunkt der Debatte über die zukünftige Mediengesellschaft stehen.' Durch die Verbindung von Telekommunikation und Computertechnik sind neue Computernetze entstanden, die es möglich machen, Botschaften nicht nur von unbegrenzt vielen Teilnehmern zu empfangen, sondern auch an ebenso viele zu verschicken. Die Nutzer erhalten die Möglichkeit, trotz räumlich-zeitlicher Trennung miteinander so zu kommunizieren, als ob sie sich in einem Dialog befänden. Vor allem das $\mathbf{I n}$ ternet hat in den letzten Jahren zu teilweise abenteuerlich wirkenden Spekulationen über bevorstehende bzw. bereits im Gang befindliche Umbrüche

${ }^{1}$ Einführungen in diese Debatte geben Williams/Pavlik (1994); Bollmann (1995); Riehm/Wingert (1995); Rötzer (1995); Bollmann/Heibach (1996); Kleinsteuber (1996). Bezeichnenderweise wird inzwischen auch in der KI-Forschung von „Verteilter Künstlicher Intelligenz" gesprochen, womit vor allem auf die Bedeutung von Kommunikation bzw. Kooperation für das Zustandekommen von Intelligenz aufmerksam gemacht werden soll (vgl. Florian 1996). in den Bereichen der Kultur und Politik moderner Gesellschaften angeregt. Die Erwartungen reichen hier von der Entstehung „virtueller Gemeinschaften“ über die Bildung „elektronischer Gegenöffentlichkeiten" bis hin zur ,digitalen Demokratie". ${ }^{2}$ Wie diese neuen Angebote und Deutungen aus sozialwissenschaftlicher Sicht zu beurteilen sind, darüber herrscht derzeit noch große Unsicherheit. Was jedoch auffällt, ist, daß in einem Großteil der aktuellen Literatur zum Thema 'neue elektronische Medien' zwei Vorstellungen gefolgt wird, die im folgenden einer genaueren Überprüfung unterzogen werden.

$\mathrm{Da}$ ist zunächst die immer wieder anzutreffende Idee einer Revitalisierung dialogischer Kommunikationsstrukturen durch die elektronischen Netze. Demnach läßt sich vor allem die Funktionsweise und kulturelle Bedeutung des Internet in Analogie zu natürlichen Prozessen der Beziehungsherstellung und Gemeinschaftsbildung begreifen. Der Gebrauch von Dialog- und Gemeinschaftsmetaphern suggeriert, daß die Interaktion in diesem neuen Medium in ähnlicher Weise sich begreifen läßt wie die vertraute Situation der face-to-faceKommunikation. ${ }^{3}$ Daß Computernetze etwas bewirken können, was natürliche Prozesse der Interaktion überschreitet, wird allenfalls durch die Hinweise auf zeit- und raumneutralisierende Effekte der neuen Netze eingeräumt, nicht jedoch als ihr primäres Unterscheidungsmerkmal verstanden.

${ }^{2}$ Einen Überblick über diese Diskussionen geben Buchstein (1996) und Hagen (1996). Siehe dort auch die weiterführende Literatur.

${ }^{3}$ Um nur einige Autoren zu nennen: vgl. Rogers (1986); Rafaeli (1988); Rheingold (1994); Goertz (1995); Hoffmann (1996); Höflich (1996). 
Deutlich läßt sich dies am Begriff der „Interaktivität" nachvollziehen. Seine Definition sowie die Frage, inwieweit die neuen Medien sich unterschiedlichen Interaktivitätsniveaus zuordnen lassen, richten sich am Vorbild reziproker Kommunikationsbeziehungen zwischen anwesenden Personen aus. Ähnlich wie die Künstliche IntelligenzForschung sich durch die Nähe ihrer Maschinen zum natürlichen Vorbild zu legitimieren versuchte. so orientiert sich auch die Diskussion über die gegenwärtig technisch fortgeschrittenste Form künstlicher Kommunikation paradoxerweise am technikfreien Ursprungsmodell wechselseitiger Bezugnahme von Personen, um anschließend - trivialerweise - festzustellen, daß die meisten, werbewirksam als interaktiv beschriebenen Angebote diesen Titel noch nicht verdienen (vgl. Goertz 1995; Riehm/Wingert 1995).

Diese Ernüchterung - ebenfalls vergleichbar den Enttäuschungen in der KI angesichts des Scheiterns ihrer ursprünglichen Leitbilder -hält jedoch nicht davon ab, die Akzeptanz der neuen Medien davon abhängig zu machen, inwieweit es gelingt, natürliche Interaktion medientechnisch zu simulieren - und damit vor allem jene kommunikationstechnischen „Fehlentwicklungen“ zu korrigieren, die aus der Sicht vieler Medientheoretiker Kultur und Öffentlichkeit unseres Jahrhunderts zu ihrem Nachteil geprägt haben. So wird - und hier handelt es sich um die zweite Basisannahme im Diskurs über die neuen Medien - von den neuen Kommunikationsmedien erwartet, daß sie vor allem die „Einbahnstraßen“ herkömmlicher Massenkommunikation in nutzerfreundliche Datenautobahnen mit "Zwei-Wege-Kommunikation“ transformieren werden. ${ }^{4}$ Die technische Unterbrechung der Beziehung zwischen Sender und Empfänger soll aufgehoben werden, um so den Nutzer aus dem Korsett einer passiven Rezipientenrolle $\mathrm{zu}$ befreien und damit seine massenmedial erzwungene Vereinzelung und Anonymität zu beseitigen.

Im Folgenden wird zu zeigen versucht, daß Entwicklungs- und Nutzungsengpässe interaktiver Medien nicht erst dort beginnen, wo es darum geht, das kommunikative Verhältnis zwischen Personen medientechnisch zu restituieren. Bereits der Versuch, Programme der Massenmedien (z. B. 'Information' und 'Unterhaltung') auf Zwei-WegeKommunikationssysteme, wie sie vor allem das Internet bereitstellt, umstellen und damit das System der Massenkommunikation zumindest teilweise

\footnotetext{
${ }^{4} \mathrm{Vgl}$. dazu die Beiträge in Kleinsteuber (1996).
}

ersetzen zu wollen, wird ohne Erfolg bleiben. Aus diesem Grunde sind nicht nur medienkritische Vorstellungen über die Massenmedien zu revidieren, sondern ist auch die Funktionalität interaktiver Medien neu auszuloten. Im weiteren Verlauf dieser Arbeit wird es deshalb vor allem darum gehen, eine angemessene Beschreibung der Massenkommunikation zu finden, um daran anschließend das gegenwärtig dominierende Bild der interaktiven Medien zu korrigieren. Es soll deutlich werden, daß die neuen Medien keine „besseren“ Massenmedien sind, von ihnen also keine Ablösung, vielmehr eine Ergänzung der Massenkommunikation und damit eine weitere Differenzierung des Mediensystems zu erwarten ist. ${ }^{5}$ In den weiteren Abschnitten wird zunächst - entgegen den medienkritischen Annahmen - die These entfaltet, daß Zeitungen, Radio oder Fernsehen sich als „Kooperationsmedien“ (Gieseke 1992) beschreiben lassen, die ihre kommunisierende Funktion paradoxerweise erst dadurch entfalten, daß sie von den Teilnahmeverpflichtungen reziproker Interaktionsformen befreien. Folgt man diesem Medienverständnis, dann lassen sich, so die weiterführende Argumentation, die spezifischen Merkmale der neuen elektronischen Medien auch nicht mehr, wie von den „digitalen Chiliasten“ (Matejovski 1996) immer wieder suggeriert, in einer Art Rückschau auf vormediale Kommunikationsformen, nämlich am Modell der mündlichen Rede festmachen. Stattdessen müßte „Interaktivität“ als strukturelle Überbietung bereits vorhandener Medienformationen rekonstruiert werden. ${ }^{6}$

\footnotetext{
5 Im Folgenden beziehe ich mich vor allem auf Arbeiten von Luhmann $(1971 ; 1981 ; 1990 ; 1992 ; 1996)$, Meyrowitz (1990), Giddens (1995), Schmidt $(1994 ; 1994 a ; 1995)$ und Spangenberg $(1988 ; 1991 ; 1996)$, die sich $\mathrm{zwar}$ vom theoretischen Ansatz her unterscheiden, jedoch darin übereinstimmen, die technische Unterbrechung zwischen Sender und Empfänger im Vergleich zur Interaktion unter Anwesenden nicht ausschließlich als Verlust, sondern auch als Gewinn kommunikativer Freiheiten zu begreifen.

${ }^{6}$ Eine weitere These des vorliegenden Beitrags ist deshalb, daß ein Großteil der Faszination, die von der Vision einer Modernisierung der Gesellschaft durch die neuen Kommunikationsmedien ausgeht, sich dem Vergessen wichtiger Funktionen verdankt, die die Massenkommunikation für die Öffentlichkeit und das Leben eines jeden einzelnen hat. Bemängelt wird deshalb auch nicht, daß Aussagen über die neuen Medien oft sehr vage und spekulativ wirken - der vorliegende Text ist selbstverständlich keine Ausnahme, auch er bewegt sich stellenweise auf der Ebene von wenig gesicherten Vermutungen. Da zum gegenwärtigen Zeitpunkt niemand wissen kann, welche Netzstrukturen und Nutzungsformen sich langfristig
} 


\section{Aufbruch in die interaktive Medienkultur}

Der Charme der neuen Medien scheint vor allem in ihrer Fähigkeit zur Kombination bislang getrennter, auf verschiedene Medien verteilter Funktionen zu liegen. Massen- und Individualkommunikation werden zusammenwachsen, stellt Fabris (1985:130) fest, so „daß die Massenmedien auch innerhalb des Sektors der technisch vermittelten Kommunikation nur einen Teilbereich abdecken und die technische Weiterentwicklung eher in Richtung Individualkommunikation - bzw. ihrer Ersetzung - geht." Lassen sich doch bereits Fernbedienung und Videorecorder als Vorstufen einer Individualisierung und Interaktivierung der $\mathrm{Me}$ dien und als Hinweis auf das unterdrückte Bedürfnis des Zuschauers nach autonomer Programmgestaltung verstehen (vgl. Hickethier 1994: $295 \mathrm{ff}$.). Durch den Einsatz dieser audiovisuellen Speichermedien wurden erstmalig auf massenhafte Weise die starren Programmschemata der Massenmedien individualisiert und den zeitlichen und inhaltlichen Vorstellungen der Rezipienten angepaßt. Solche Ansätze einer Individualisierung der Auswahl von Medienangeboten und des Eingreifens in den Ablauf der Medienkommunikation auf der Rezipientenseite („de-massification“) werden unterstützt durch die Strategien einer Spezialisierung der Angebote auf der Anbieterseite. An die Stelle des konventionellen Medienangebots an ein unspezifiziertes Publikum (,Einer-an-Viele-Medien") treten zunehmend Medienverbundsysteme, die sich mit ihrer Angebotsvielfalt an spezielle, homogene Gruppen richten und dementsprechend individualisiert und diversifiziert sein müssen („Viele-an-Einen-Medien“). Dieser Trend zur Verspartung zeichnet sich bereits bei den Massenmedien ab: „Aus der Sicht der Fernsehprogrammanbieter entspricht der individuellen genrespezifischen Rezeption von Programmteilen nach individuellen Zeitmustern und in selbstgewählten Sozialformen, die in Entertainment- und Kaufaktivitäten integriert sind, die riskante Suche nach dem Fernsehpublikum vor dem Bildschirm. Individualisierte Mediennutzung führt dazu, daß das Ge-

durchsetzen werden, ist dies ein nicht zu umgehendes Manko. Kritisiert wird vielmehr der für viele derzeit hoch gehandelten Medientheoretiker charakteristische Verzicht auf vergleichsweise gesicherte Kenntnisse uber die "alten Medien“, mit denen sich Vermutungen darüber anstellen lassen, welche kommunikativen Funktionen von den neuen Medien nicht ubernommen werden können. samtpublikum sich auflöst und nur individuell, also entsprechend ihrer Nutzungsmuster, Stile und Präferenzen, zu adressieren ist" (Bachmair 1996: 285). Ähnlich wie sich beispielsweise ein an unterschiedliche Adressatengruppen orientierter Zeitschriftenmarkt entwickelt hat, wird nun auch für Radio und Fernsehen eine Entwicklung zu „Quotenmedien“ (Schulze 1995) und definierten Rezipientengruppen mit nicht-verallgemeinerungsfähigen Interessen prognostiziert: „Differenzierungs- und Integrationsprozesse umspannen das telekommunikative Massenkommunikationssystem, das von verteilten und breitgestreuten Angeboten mit universellen Inhalten bis zu Spezialprogrammen für klar eingegrenzte Zielgruppen reicht" (Mast 1986: 180). Die Anonymität der Massenkommunikation wird durch diesen Trend zu personell und/oder regional klar definierten Empfängerkreisen bzw. Zugangschancen langfristig abgeschwächt. Das unpersönliche Massenpublikum verwandelt sich allmählich in klar abgegrenzte Nutzergruppen (,special-interest-groups“). Zusätzlich zur Verspartung und Segmentierung der traditionellen Vollprogramme kommen Pay-TV (Abonnement gesamter Programme gegen Gebühr), Near-Video-on-demand (zeitversetzte Ausstrahlung gleicher Programmteile) bis hin zu Video-on-demand oder Pay-per-view, wo der Nutzer aus einer Programmdatenbank ein einzelnes Angebot abruft und dafür eine Gebühr zu entrichten hat. ${ }^{7}$ Zusammen mit den Angeboten der Online-Kommunikation gewinnt der Nutzer ein immer breiteres Repertoire an Angeboten zur „... Befriedigung individueller Bedürfnisse hinsichtlich des Abrufs von Informationen (Daten, Texte), des Kommunizierens mit Organisationen, Behörden, Institutionen (Auskunft, Bestellung von Waren und Diensten) und der Selbstbedienung (Herstellung eigener Filme, Kauf und Leihen von Videobändern und -platten)“ (Ronneberger 1982: 17). All diese Medienangebote verbindet, da $B$ sie ,... dem Rezipienten Gestaltungsfunktionen zuweisen, d.h. Rückmeldungen aller Art, seien es nun Zugriffsmodalitäten oder der technischen Kapazität der Rückkanäle entsprechend Mitteilungen in Ja-nein-Form bzw. Text-, Bild- oder Sprachkommunikation" (Mast 1986: 185). Die Hauptattraktion der neuen Medienwelt sind jedoch die elektronischen Kommunikationssnetze. Dazu zählen die traditionelle Mail-Box-Szene, Online-Dienste ebenso wie die

\footnotetext{
${ }^{7}$ Einen Überblick uber die technische und organisatorische Vielfalt dieser neuen Medien geben z.B. Faulstich (1994), Kubicek und Seeger (1993) und Seeger (1995).
} 
Vielzahl von Kommunikationsdiensten im Internet. Erst hier findet offenbar der Wechsel von der vertrauten „Einer-an-Viele-Kommunikation“, wie sie sich mit den herkömmlichen Massenmedien verbindet, zu einer ,Viele-an-Viele-Kommunikation“ statt. ${ }^{8}$ Sog. Computerforen, Newsgroups, Mailgroups und andere interaktive Kommunikationsdienste des Internet bieten ihren Teilnehmern die Gelegenheit, in einen Gedankenaustausch zu treten, indem ,nicht nur auf die vorgängige Botschaft, sondern auch auf noch weiter davorliegende kommunikative Sachverhalte eingegangen wird und damit ein gemeinsamer Sinnhorizont eröffnet wird“" (Höflich 1996: 63). Es entsteht so eine Situation aktualer Reziprozität, da die Teilnehmer im Verlauf der Kommunikation - vergleichbar dem Sprecher/Hörer Wechsel in natürlich-sprachlichen Situationen - immer wieder ihre Sender-/Empfänger-Positionen wechseln können (vgl. Rafaeli 1988: 119). Deshalb sind es vor allem die Kommunikationsdienste in den Netzen, die nach Meinung kritischer Beobachter die Bezeichnung 'interaktiv' verdienen. ${ }^{9}$

Es kann nicht verwundern, wenn Massenkommunikation in den Szenarien dieser neuen interaktiven Medienwelt keine Berücksichtigung findet. Sie behindert nur den Weg in eine ,basisdemokratische" Kommunikationsordnung, in der es eine strikte Trennung zwischen Sender und Empfänger nicht mehr geben wird. Es ist vor allem ihre $A$ nonymität, nämlich Texte, Bilder oder Töne zu verbreiten, die an ein nicht näher spezifiziertes Publikum gerichtet sind, und ihre Einseitigkeit, die den Rezipienten auf die Rolle eines passiven und distanzierten Beobachters reduziert, was Zeitungen, Radio und Fernsehen in ihrer traditionellen Form vor dem Hintergrund der durch Computertechnik bereitgestellten Optionen, Medienkommunikation $\mathbf{z u}$ interaktivieren, fragwürdig werden läßt. Beide Eigenschaften werden dafür verantwortlich gemacht, daß in der Massenkommunikation der Rezipient zum vereinzelten, kommunikationsunfähigen „Empfänger“ degradiert wird und keinen Spielraum für die Entfaltung seiner persönlichen Informations-und Unterhaltungsbedürfnisse erhält. Besonders das Fernsehen mit seiner starren Beziehung zwischen Sender und Empfănger, mit seinen festgelegten Senderhythmen und Pro-

${ }^{8} \mathrm{Vgl}$. die Beträge in Bollmann/Heibach (1996).

${ }^{9}$ Hilfreiche Unterscheidungen zwischen verschiedenen "Interaktivitätsniveaus" neuer elektronischer Medien finden sich bei Riehm/Wingert (1995: 68 ff.). Vgl. auch die Beiträge in Zacharias (1996). grammstrukturen, wird nun zum Inbegriff eines „tyrannischen“ Mediums (vgl. Flusser 1995; Rheingold 1995).

Dieses Verständnis der Massenmedien dient der Profilierung der neuen Medien als Kontrastfolie: ${ }^{10}$ Kaum jemand bezweifelt, daß es zukünftig immer weniger Zuschauer oder Leser geben wird, die sich freiwillig den Programmdiktaten der Massenmedien beugen und damit zufrieden geben werden, vorfabrizierte Inhalte zu festen Zeitpunkten zu konsumieren. Stattdessen, so wird vermutet, wird ein eher spielerisch-interaktiver Umgang mit den Medien dominieren (vgl. Krämer 1995). In Zukunft wird der Verbraucher seine Bereitschaft, den Medien Aufmerksamkeit zu widmen, davon abhängig machen, inwieweit deren Angebote seinen persönlichen Interessen entgegenkommen (vgl. Rötzer 1996). Er wird danach verlangen, frei von jeglicher physikalischer Beschränkung kommunizieren zu können, und aus einem immer reichhaltigeren Medienangebot auswählen wollen. Wennmöglich wird er sich selbst an der Herstellung der Medienangebote beteiligen (vgl. Negroponte 1995:190ff.). Der Nutzer einer sich immer weiter entgrenzenden Informations- und Kommunikationswelt ist nicht mehr Konsument und Abnehmer vorfabrizierter Produkte, sondern wird als Entscheider und Kommunikator in mediale Prozesse einbezogen (vgl. Schulze 1995). Radikale Vordenker des interaktiven Medienzeitalters verkünden deshalb den unvermeidlichen Abstieg der Massenmedien als Leitmedien und sprechen auch schon von der „Fernseh-Dämmerung“ (Kittler 1993) und vom „Ende der Gutenberg-Galaxis“ (Bolz 1993). ${ }^{11}$

Was in der Tradition linker Medienkritik immer wieder eingeklagt wurde, nămlich die Aufhebung der Trennung von Medienprodukt und Medienrezipient, scheint heute durch elektronische Datennetze und interaktives Fernsehen in unmittelbare Reichweite gerückt worden zu sein. In der gedanklichen Figur einer (inter-)aktiven und selbstverantwortlichen Mediennutzung gehen die offensiven Verfechter der neuen Medien eine auffällige Allianz mit den Kritikern der „alten“ Massenmedien

${ }^{10}$ Eine im ubrigen nicht ungewöhnliche Vorgehensweise. Bereits die sozialen und kulturellen Konsequenzen der Schriftlichkeit wurden, wie Platons Kritik im „Phaidros“ an Sokrates zeigt, mit Hilfe der Differenz zu den Wirkungen der Mündlichkeit definiert. Vgl. die Interpretation des Phaidros durch Goody und Watt (1981).

11 Der Ausdruck von Kittler ist Wetzel (1995: 19) entnommen. 
ein (vgl. Maresch 1995). So bemängelte bereits Brecht (1967) die Einseitigkeit des Radios und schwärmte von einem Medium, das nicht nur verstünde auszusenden, ,sondern auch zu empfangen, also den Zuhörer nicht nur hören, sondern auch sprechen zu machen und ihn nicht zu isolieren, sondern ihn in Beziehung zu setzen." Vor und nach dem zweiten Weltkrieg waren es vor allem die Vertreter der sog. „Frankfurter Schule“, wie Horkheimer, Adorno, Benjamin und Marcuse, die in ihren Arbeiten die Industrialisierung von Kultur durch die Massenmedien anprangerten. Sie sahen in der Senderdominanz der Massenpresse und Rundfunk- und Sendeanstalten eine entscheidende Voraussetzung für die strukturelle Angleichung von Konsumenten- und Rezipientenrolle und damit "für den Blockierungszusammenhang spätkapitalistisch kontrollierten Bewußtseins" (Adorno 1963). Seinen vorläufigen Höhepunkt erreichte der intellektuelle Widerstand gegen die Massenmedien in den 70er Jahren als marxistisch orientierte Medienkritik, die die Funktionsprinzipien der Massenmedien konsequent aus der Logik kapitalistischer Warenproduktion herleitete (vgl. Holzer 1973; Prokop 1972; 1973; Heinze 1990). ${ }^{12}$ Ähnlich wie heute die Verfechter einer vernetzten Medienkultur einen Medienakteur fordern, war man sich vor allem in dieser Phase sicher, daß nur durch eine Aufhebung der Trennung zwischen Sender und Empfänger (in der damaligen Terminologie: durch eine Aufhebung der Rollenverteilung von Produzenten und Konsumenten) die entfremdenden und verdinglichenden Wirkungen der Massenkommunikation $\mathrm{zu}$ uberwinden sind (vgl. Enzensberger 1970; Janshen 1980; Prokop 1973).

Nicht erst seit heute also stimulieren Massenmedien das Nachdenken über Medien. Während jedoch die Medienkritik jener Jahre eine weitgehend innerakademische Angelegenheit blieb, bezieht der heutige Mediendiskurs seine Legitimität aus der überbordenden Fülle medientechnischer Experimente. Medien, die einem isolierten Empfänger im Takt ihrer Erscheinungs- bzw. Sendezeitpunkte bereits fertige Angebote oktroyieren, wirken deshalb nicht nur in ideologiekritischer, sondern auch in technologischer Hinsicht als überholt. Deshalb ist es unplausibel, lediglich eine Erweiterung des „alten“ Informations- und Kommu-

12 Wenngleich das Medienthema in der Soziologie in den Jahren danach eine vergleichsweise geringe Rolle spielt, wird das Kulturindustrie-Argument immer wieder an prominenter Stelle aufgegriffen. So beispielsweise von Beck (1986: 213). nikationssystem zu fordern. Einzig eine radikale „Verschiebung der Intelligenz vom Sender zum Empfänger“ (Negroponte 1995:29) durch die möglichst spurlose Transformation der Massenmedien in interaktive Medien erscheint jetzt noch sinnvoll.

\section{Virtuelle Vergesellschaftung}

Modelle, in denen von "Massenkommunikation“ die Rede ist, wirken widersprüchlich, da aufgrund der technischen Unterbrechung zwischen Sender und Empfänger zumindest der Rezipient zur Kommunikationsunfähigkeit verurteilt ist. Vor allem Merten (1977) hat die Frage aufgeworfen, ob Massenkommunikation wegen des Verzichts auf die Anwesenheit seiner Teilnehmer, seiner Anonymität und Einseitigkeit überhaupt als Kommunikation verstanden werden kann. Um als soziales Handeln zu gelten, müßte zumindest die Minimalforderung erfüllt werden, daß sich zwischen Kommunikator und Rezipienten aufeinander bezogene Erwartungen einstellen. Erforderlich wäre ein „... Kommunikator, der auf 'seine' Rezipienten hin handelt, und der Rezipient, der sich an den Vorstellungen von 'seinem' Kommunikator orientiert..." (Merten 1977: 146). Der Alltag der Massenkommunikation zeigt jedoch, „... daß vermittelte Aussagen großenteils pragmatisch frisiert und mit großen Zufälligkeiten auf den Weg gelangen, und sie zeigt andererseits nicht selten auch einen Rezipienten, der seinem Kommunikator eingeschlafen und in Filzpantoffeln gegenübersitzt" (Merten 1977: 146). Daß es sich dennoch hier um Kommunikation handelt, erschließt sich erst dann, wenn, so Merten, die Funktionen der Massenkommunikation nicht mehr ausschließlich im Rahmen der „Sender-Empfänger-Beziehung“, sondern auBerdem im Hinblick auf die Rolle der Rezipienten als Elemente eines Medienpublikums thematisiert werden. ${ }^{13}$

Fernsehen, Radio und Zeitungen formen aus zuvor getrennten Bevölkerungssegmenten und Milieus eine „virtuelle Einheit“, die ihre Identität dadurch gewinnt, daß die gleiche Information in vielen Kopien zeitgleich allen Rezipienten verfügbar gemacht wird (vgl. Meyrowitz 1990: $135 \mathrm{ff}$.). Unter

13 Diese Feststellung relativiert auch all jene Erklärungsmodelle, die die Entwicklung und Reproduktionslogik der Medien ausschlieBlich unter dem Aspekt institutionalisierter bzw. rollenförmig organisierter Beziehungen zwischen den Anbietern und Nutzern von Medienangeboten thematisieren. Beispiele sind hier Hunziker (1988) oder Kubicek und Schmid (1996). 
der technischen Voraussetzung, daß eine Aussage, ein Ton oder ein Bild unbegrenzt reproduzierbar wird, also in identischer Weise an eine prinzipiell unbegrenzte Zahl von Rezipienten verbreitet werden kann, emergiert eine öffentliche „Informationswelt" (Meyrowitz 1990: 189), die im Satellitenzeitalter transkontinentale Dimensionen erreicht. Mehr noch als Zeitungen und Radio ist das Fernsehen beteiligt an der Aufhebung physischer und sozialer Grenzen, die die fernsehlose Gesellschaft in unterschiedliche Orte und Kulturen aufteilten (vgl. Meyrowitz 1990; Großklaus 1995). Besonders dieses Medium hebt Trennungslinien zwischen privat-öffentlich und nah-fern auf und schwächt die Abhängigkeit des Einzelnen von lokal verfügbaren Informationsangeboten ab. Massenkommunikation eröffnet, so Luhmann (1981), einen thematischen Horizont, auf den sich der Rezipient wie auf eine objektiv vorgegebene Welt beziehen kann. Eine ihrer entscheidenden Funktionen liegt deshalb,$\ldots$ in der Beteiligung aller an einer gemeinsamen Realität oder, genauer gesagt, in der Erzeugung einer solchen Unterstellung, die dann als operative Fiktion sich aufzwingt und zur Realität wird" (Luhmann 1981: 320). So wie jeder, der seine Botschaft im Fernsehen oder Radio verbreitet, es nicht - wie noch bei den meisten Printmedien - mit einem begrenzten Empfängerkreis zu tun hat, sondern mit einem aus verschiedenen Segmenten der Gesellschaft sich zusammensetzenden anonymen Zuschauer- oder Hörerkreis, so weiß sich auch der Rezipient im Vollzug des Zuschauens oder Hörens durch den Akt der zeitgleichen Konsumption eines identisch gehaltenen Kommunikats verbunden mit einer ihm unbekannten Menge weiterer Rezipienten.

Der Begriff des Massenpublikums läßt sich deshalb als zu unterstellende zeitgleiche informationelle Synchronisation sämtlicher Mitglieder einer Gesellschaft deuten. Indem sie durch die Präsentation ihrer Themen eine Vorauswahl dessen treffen, was in der Gesellschaft erinnert und kommuniziert werden kann, erzeugen Massenmedien eine Art „Kollektivbewußtsein", welches dafür sorgt, daß sich zwischen den Rezipienten, die während der Medienrezeption nicht kommunizieren und deshalb anonym bleiben, eine Vorverständigung über die wichtigsten Ereignisse in der Welt einspielt (vgl. Luhmann 1971: 10; Luhmann 1996: 120; Meyrowitz 1990: 179). Sie lenken die Aufmerksamkeit einer unbegrenzten Zahl von Rezipienten auf eine eng begrenzte Zahl von Aussagen (Merten 1977: 147): Wer beispielsweise in der Zeitung eine Nachricht liest, der weiß, daß nicht nur er, sondern auch andere dieselbe Nachricht gelesen haben (könnten). Jeder Zeitungsleser weiß also, was andere Leser wissen können. Er weiß ferner, daß andere Leser wissen können, was er als Leser weiß. Und er kann sogar wissen, da $\beta$ andere wissen, da $\beta$ er weiß, was sie wissen. Die vergesellschaftende Funktion der Massenkommunikation beruht auf dieser „Reflexivität des Wissens“ (Merten 1977: 147; Joußen 1991: 155 ff.). Denn im Gegensatz zum interaktiven Kommunikationssystem, das auf der Reflexivität von Wahrnehmungen aufbaut, reproduziert sich das System der Massenkommunikation ,... durch Vorstellungen an anderen ..., so $\mathrm{da} \beta$ es hier als virtuelles soziales System gekennzeichnet werden soll, das durch Spiegelung am Kommunikator zustandekommt (Merten 1977: 150; Herv.i.O.).

Es fällt schwer sich vorzustellen, wie anders als durch Massenkommunikation eine überregionale, mitunter sogar transnationale „Sofort-Integration“ (Luhmann 1981) unter der Bedingung funktional differenzierter Gesellschaften zu erreichen ist. Gesellschaften dieses Typs sind zum einen mit dem Problem einer ungewöhnlich hohen Kontingenz des kommunikativ Möglichen konfrontiert. Beschreibungen sozialer Komplexität oder natürlicher Sachverhalte verlangen deshalb immer unterscheidungsabhängige und damit zwangsläufig selektiv operierende Beobachtungsinstrumente und -verfahren sowie Institutionen, die von ihnen Gebrauch machen. ${ }^{14}$ Aufgrund ihrer internen Vielfalt von eigensinnigen Beobachterperspektiven und Wirklichkeitsentwürfen erzeugen sie auBerdem einen Bedarf für Sinnstrukturen gesamtgesellschaftlicher Verständigung (vgl. Marcinkowski 1993: 119). Damit Kommunikation möglich wird, in der Gesellschaft sich selbst vergegenwärtigt, bedarf es folglich nicht nur Mechanismen der Komplexitätsreduktion, sondern ferner der Einfuihrung von Regeln, die diese Reduktion im Sinne einer Überbrückung systemspezifischer Kommunikationsstrukturen ermöglicht.

Die Massenmedien leisten diese erforderliche Komplexitätsreduktion, indem sie eingrenzen, was Gegenstand von Kommunikation werden kann. Ihre vornehmliche Funktion liegt in der Auswahl und Publikation kontingenzlimitierender Selektionen (Themen), die einem anonymen Publikum als realer gemeinsamer Bezugspunkt ihrer Alltagskommunikation dienen. Auf diese Weise ermögli-

14 Gieseke (1992: $286 \mathrm{ff}$ ) spricht in diesem Zusammenhang von einer "Modellabhăngigkeit" der Kommunikation. 
chen sie „... die Beobachtung der Gesellschaft durch die Gesellschaft" (Gerhards 1994: 86). Die Engführung öffentlicher Kommunikation durch die Unterstellung gemeinsamer Themen ist so gesehen - trotz aller Kritik an einseitiger Berichterstattung in den Medien - unvermeidlich. Ohne die Massenmedien wäre die Gesellschaft, so zuletzt Luhmann (1996), in einem grundsätzlichen Sinne nicht beobachtbar und kommunizierbar und damit nicht reproduktionsfähig. Erst sie entwickeln die dafür erforderliche Abstraktionslage und $\mathrm{Ge}$ schwindigkeit (vgl. Giddens 1995: 101). Eine Aufmerksamkeitssteuerung im Sinne lebensweltlicher Selbstverständlichkeiten wäre mit dieser Aufgabe uberfordert, weil sie zu konkret und einseitig wäre, um der gerade fur moderne Gesellschaften charakteristischen Vielfalt der Milieus und Lebensstile einen gemeinsamen akzeptablen thematischen Bezugspunkt zu liefern. Deshalb scheiden die Sinnstrukturen von Personen, Familien oder Milieus als Basis einer solchen Selektion aus. Erst ein die lokalen Verständigungshorizonte einzelner Milieus und Kollektive überschreitendes System von Prämissen der Komplexitätsreduktion besitzt die Abstraktheit, die für eine „Sofort-Verständigung“" der sozialen Systeme moderner Gesellschaften erforderlich ist. Nur das System der Massenkommunikation ist in der Lage, Themen auf eine Weise auszuwählen, ,die sie zugleich virtuell für alle Mitglieder der Gesellschaft qualifiziert, die sie mehr oder weniger 'öffentlich' verbindlich, verallgemeinerungsfähig macht" (Böckelmann 1975: 45, Herv.i.O.). Ein solches System schafft Öffentlichkeit im Sinne von Kommunikationssituationen, die nicht durch partikulare Aufmerksamkeitsregeln eines Teilsystems der Gesellschaft, sondern eben durch Themen vorstrukturiert werden, „... bei denen universelle Akzeptiertheit vorausgesetzt werden kann" (Luhmann 1971: 22). Öffentlichkeit heißt, die Bekanntheit eines Themas voraussetzen zu können. Öffentliche Themen sind deshalb nur schwer zu umgehen. Wer sie ständig ignoriert, setzt seinen Status als zurechnungsfähiges Mitglied einer Gesellschaft auf's Spiel.

Die Bedeutung der Massenmedien als Selektionsmechanismus beschränkt sich nicht auf das System der Gesellschaft, sondern zeigt sich auch auf der Ebene seiner Teilsysteme, wie sich an der Komplementärbeziehung zwischen der öffentlichen Meinung und dem System der Mehrparteiendemokratie beispielhaft darstellen läßt (vgl. Luhmann 1971, 1990, 1992; Marcinkowski 1993; Gerhards 1994). Das Kardinalproblem sozialer Systeme, Strukturen einer sinnhaften Erfassung und Reduktion ei- ner überkomplexen Umwelt zu finden und zu stabilisieren, stellt sich für die Politik im Hinblick auf die prinzipiell unerschöpfliche Vielfalt politikfähiger Themen. Um nicht in einer Anspruchsflut zu ertrinken, dient hier die öffentliche Meinung als ein Mechanismus der Steuerung von (knapper) Aufmerksamkeit, indem sie einerseits aus der Fülle öffentlich kommunizierter Themen die Menge politikrelevanter Themen (,issues") filtert und so für eine inhaltliche Vorverständigung politischer Kommunikation sorgt, gleichzeitig - im Sinne 'loser Kopplung' - offen läßt, wie mit diesen Themen und Problemen im politischen Kontext verfahren wird: „Öffentliche Meinung ist gleichsam substantivierte politische Kontingenz - ein Substantiv, dem die Lösung des Problems der Reduktion der Beliebigkeit des rechtlich und politisch Möglichen anvertraut" (Luhmann 1971: 10). Auch diese Selektionshilfe leisten in modernen Demokratien die Massenmedien, die durch Themenvorgabe (,agenda-setting“) „... ein gemeinsames Sichbeziehen auf identischen Sinn (ermöglichen) und ... das Aneinandervorbeireden (verhindern)" (Luhmann 1971: 13). Nicht jedes Medien-Thema hat folglich politische Relevanz, allerdings muß (fast) jedes Thema, das in der Politik Resonanz finden soll, die Aufmerksamkeitsfilter der öffentlichen Meinung passieren. Deren Funktionsfähigkeit bemißt sich folglich auch nicht an der Vernünftigkeit oder Konsensfähigkeit kommunizierter Meinungen, „sondern an der Form der Themen politischer Kommunikationen, an ihrer Eignung als Struktur des Kommunikationsprozesses. Und diese Funktion besteht nicht in der Richtigkeit der Meinungen, sondern in der Unsicherheit absorbierenden, Struktur gebenden Leistung von Themen" (Luhmann 1971: 15). Luhmann zufolge richtet sich deshalb die für eine Anpassung an eine (teilweise) widersprüchliche Umwelt erforderliche Variation politischer Kommunikation (u.a.) nach der Themenmobilisierungskapazität der Massenmedien. ${ }^{15}$ Politik schützt sich also vor Überlastungen, indem sie auf die Filterwirkung setzt, wie sie durch die „politische Meinung“ im Sinne verallgemeinerungsfähiger Themen erzeugt wird. Ohne thematische Restriktionen als Bezugsgrößen drohte das politische System orientierungslos zu werden und sich zur Entscheidungs- und Handlungsunfähig-

15 Hiermit wird gegenüber stärker normativen Modellen sicherlich ein vergleichsweise anspruchsärmeres Konzept der Offentlichkeit und öffentlichen Meinung behauptet. Kritisch dazu Neidhardt (1994) und Peters (1994). Für den vorliegenden Begründungszusammenhang ist diese Feststellung jedoch ohne Belang. 
keit $\mathrm{zu}$ verurteilen. Politik wird deshalb erst dadurch im Sinne von politischen Positionen und Angeboten für sich selbst und für den Bürger beobachtbar, soweit die Parteien ihrerseits die Möglichkeit haben, im Medium der öffentlichen Meinung auf eine angemessene Weise über die Präferenzen der Bürger und Angebote ihrer Konkurrenz sich zu informieren (vgl. Gerhards 1994).

Damit nun der Eindruck einer gemeinsamen thematischen Referenz zustande kommt, dürfen die Programmstrukturen der Massenmedien und die durch sie vermittelten Wahrnehmungs- und Deutungsformen als spezielle Weltzugänge erst gar nicht in Erscheinung treten - die Selektivität medialer Darstellungstechniken hat latent zu bleiben (vgl. Schmidt 1994a). Die Funktion von Funk und Fernsehen, dem Rezipienten das Gefühl zu geben, als Mitglied einer großen Gemeinschaft den wichtigsten Ereignissen in der Welt unmittelbar beizuwohnen. ist also davon abhängig, da $\beta$ der Herstellungsproze $\beta$ der Medieninhalte unbeobachtet bleibt. Denn nur solange, wie die Medienangebote nicht als kontingente, an medieninternen Kriterien sich orientierende Entscheidungen und als Mitteilungen eines Senders thematisiert werden, bleibt die Illusion erhalten, daß alle Leser bzw. Zuschauer sich auf eine identische Information beziehen. Dieser Fiktion erliegt vor allem der Fernsehzuschauer, weil im Gegensatz zu sprachlichen Äußerungen, die eindeutig als Kommunikationsleistung identifiziert werden, visuelle Wahrnehmungsgegenstände kaum mehr als Erzeugnisse selektiver Beobachtung wahrgenommen werden. Wahrnehmungsprozesse erschweren die Unterscheidung zwischen Inhalt und Darstellung, so daß besonders beim Fernsehen, dessen Darstellungstechniken natürlichen Sehgewohnheiten sehr nahekommen, der Eindruck gewonnen wird, die gezeigte Welt ließe sich gar nicht anders als in der aktuellen Form darstellen (vgl. Spangenberg 1988: 784).

Dies erklärt, warum beispielsweise im Bereich der Information, vor allem in Nachrichtensendungen, auf mediale Inszenierungen, die die Kommunikationsabhängigkeit und Selektivität der Inhalte hervorheben würden, weitgehend verzichtet wird. Nur so wird die in den Massenmedien präsentierte Welt als eine von Interessen und Meinungen unabhängige, gleichsam nicht-kommunizierte, objektive Welt registriert. Fernsehbilder oder Zeitungsmeldungen erwecken den Eindruck, als stünden sie in einem objektivierenden Verhältnis zu den von ihnen berichteten Ereignissen. Sie machen uns glauben, die Gesellschaft lasse sich von außen beobachten und zwar durch die Setzung einer Gren- ze zwischen Medien und Gesellschaft, obwohl doch auch die Medien Teil dieser Gesellschaft sind. Durch die Fokussierung auf Themen wird die Unübersichtlichkeit bzw. Unbeobachtbarkeit gesellschaftlicher Komplexität zur „anderen“, abgedunkelten Seite latent bleibender Aufmerksamkeitsregeln. Die Gesellschaft taucht in der Massenkommunikation deshalb immer nur als ein nach Maßgabe kognitiver Schemata reduzierter Sachverhalt in den Programmbereichen von 'Unterhaltung', 'Information' oder 'Werbung' auf (vgl. Schmidt 1994: 172; Luhmann 1996: 196 ff.). Auf eine Reflexion dieser Selektivität wird in den Medien weitgehend verzichtet. Stattdessen herrscht dort eine gewisse Betriebsblindheit hinsichtlich der Konstruktionsabhängigkeit ihrer Beschreibungen und Kommunikationsangebote, die ihren nachhaltigsten Ausdruck im ritualisierten Bekenntnis zu objektiver Berichterstattung findet. Eine Thematisierung der Unterscheidungabhängigkeit und Selektivität der Medienrealitäten hätte zur Folge, daB die Unbeobachtbarkeit der Gesellschaft als ein für alle Beobachtung und Kommunikation ermöglichender Raum zur Gewißheit würde. Es ließe sich dann kaum mehr leugnen, daß auch die Realität der Massenmedien auf kontingente kognitive Voraussetzungen angewiesen ist. Medien gelten deshalb nur solange als Wirklichkeitsvermittler, wie die Gesellschaft als „,andere Seite" der präsentierten Themen unsichtbar bleibt. Ebenso würde eine Einmischung der Rezipienten in die Auswahl und Darbietung der Themen die Fiktion der Wirklichkeitsvermittlung empfindlich stören, da hierdurch die Aufmerksamkeitsregeln auf partikulare Interessen heruntergebrochen würden. Persönliche und damit nicht-verallgemeinerungsfähige Botschaften gefährden die Integrationsfähigkeit der Medien, da sie lediglich den Erwartungen konkreter Rezipientengruppen entgegenkommen. Massenmedien erfüllen deshalb ihre integrative bzw. vergesellschaftende Funktion nur unter der Bedingung des Interaktions- bzw. Kommunikationsverzichts seitens ihrer Rezipienten.

Tatsächlich interessiert sich der Zuschauer oder Zuhörer für das gesendete Ereignis, nicht dafür, warum das Ereignis gesendet wurde. Der Reiz der Massenmedien liegt in der Teilhabe an einer von persönlichen Perspektiven und selektiven Entscheidungen unabhängigen objektiven Welt. Man sieht, was andere sehen, man ließt, was andere lesen. Zuschauer interessieren sich kaum für die Motive, Interessen und Sichtweisen der Medienproduzenten als vielmehr dafür, wie eine Informa- 
tion auf andere Empfänger gewirkt hat (vgl. Merten 1977: 151). Massenmedien lassen sich deshalb, so Schmidt (1994a: 16) in Anlehnung an Luhmann, als "Medien erster Ordnung" beschreiben: Sie erzeugen auf der Rezipientenseite die Illusion einer Direktwahrnehmung, obwohl sie tatsächlich Kommunikatoren spezifischer Wirklichkeitsansichten sind. Besonders das Fernsehen verhindert aufgrund der Verbreitung quasi-objektiver (Sinnes-)Daten, das Dargestellte als Selektions- bzw. Kommunikationsleistung zu begreifen, ebenso wie es erschwert, sich als Zuschauer von der eigenen Wahrnehmung zu distanzieren und die eigene Rezeptionsleistung zu vergegenwärtigen (vgl. Spangenberg 1988).

\section{Massenkommunikation und Individuum}

Der Vorwurf einer Vereinzelung der Menschen durch den Konsum der Massenmedien scheint also nicht haltbar zu sein. Und die Befürworter interaktiver Medien müssen sich fragen lassen, wie das ebenfalls von ihnen beschworene Gemeinschaftsgefuhl (unter Fremden!) anders als durch massenkommunikative Inklusion der Rezipienten erzeugt werden soll. Doch wie steht es mit dem Vorwurf der autonomie- und persönlichkeitsverletztenden Wirkungen dieses Kommunikationstyps?

Zwar gewinnen Ereignisse als Themen in den Massenmedien einen Bekanntheitsgrad, der jeden geradezu auffordert, sie zur Kenntnis zu nehmen. Auch erzeugen Medien durch ihre Themenselektion eine gewisse Vororientierung und Kanalisierung der Kommunikation. Durch die Unterbrechung des unmittelbaren Kontakts zwischen Sender und Empfänger bleibt jedoch unbestimmt, wie die Angebote aufgenommen werden. Massenmedien produzieren zwar ständig neue Anlässe für Kommunikation, sie erzeugen jedoch, wie vor allem in der sog. Diffusionsforschung nachgewiesen wurde (vgl. Hunziker 1988: 108), keinen Konsensdruck, sondern lassen offen, welchen Verlauf diese nimmt: Jeder hat nicht nur die Möglichkeit, aus dem Angebot auszuwählen, was ihn interessiert, er kann auch einzelnen Aussagen widersprechen, ja er muß sogar die angebotenen Botschaften interpretieren, diese vor dem Hintergrund seiner Zielund Wertvorstellungen mit Bedeutung versehen und in alltägliche Sinn- und Handlungszusammenhänge übersetzen (vgl. Dervin 1983; Renkstorf 1989; Krotz 1991; Krotz 1995). Massenkommunikation verläuft deshalb zwar anonym, ihre Themen bilden jedoch, wie in der Rezeptionsfor- schung seit langem bekannt ist, den Gegenstand anschließender Diskussion und Meinungsbildung und sind deshalb immer auch Kommentierungen und Bewertungen unterworfen: „Zuschauer reden beim Fernsehen und nach dem Fernsehen nicht selten über den 'Fernsehtext'. In unterschiedlichen alltäglichen Kontexten werden die Fernsehereignisse kommentiert, diskutiert, erzählt und auf vielfache Weise gedeutet und umgedeutet. Was der Fernsehtext anbietet, wird so nach den Bedürfnissen und Mustern der Zuschauer kommunikativ angeeignet" (vgl. Holly und Püschel 1993: 7).

Die Entwicklung der Massenmedien und mit ihr die Evolution anonymer Kommunikationsformen hat weder zu einer Abschaffung persönlicher Meinungsbildung, noch zu einer Verdrängung natürlicher Interaktion geführt. „Interaktive Kommunikation und Massenkommunikation", so stellt Schmidt (1994: 64) in Anschluß an Merten (1977) fest, „beziehen sich aufeinander und partizipieren aneinander: Man spricht über das Fernsehen, und das Fernsehen spricht, worüber man spricht. Mit anderen Worten, unvermittelte Kommunikation kann sich den Aussagen der 'Massenkommunikation' überlagern und deren spezifische Bewertung hervorbringen."Interpersonale Kommunikation erfährt durch das System der Massenmedien keinen Funktionverlust, sondern einen Funktionswandel bzw. -zuwachs, insofern sie von der reinen Vermittlung von Informationen entlastet und nun stärker der Reflexion und Rekontextualisierung massenmedialer Selektion dient (vgl. Merten 1977; Schenk 1991; Charlton 1993). Nur die nicht-vermittelte Interaktion besitzt das notwendige Tempo und die erforderliche Geschmeidigkeit, um die anonymen Themen der Massenmedien in die persönlichen Erfahrungskontexte und von gruppen- bzw. milieuspezifischen Normen und Werten geprägten Handlungssituationen der Rezipienten einzubinden.

Kommunikative Interaktion ist - mit Giddens (1995) gesprochen - ein Mechanismus der „Rückbettung“ der Selektionen, die zuvor unter der Bedingung "entbettender" Massenkommunikation von einem anonymen Publikum rezipiert wurden. Hieraus läßt sich die These ableiten, daß erst durch Massenkommunikation die für moderne Gesellschaften charakteristische Kopplung von Prozessen der Anonymisierung und Individualisierung bzw. Reflexivierung sozialer Lebenslagen möglich ist. Unterscheidungsmöglichkeiten und Individualisierungschancen gehen Hand in Hand mit medientechnisch forcierter Vermassung, Entkontextualisierung und Globalisierung, ja werden sogar hierdurch gesteigert! Vor allem Giddens 
(1995: 102) sieht in der Verwobenheit von Entfernung und Nähe, von Integration und Abgrenzung, von Massen- und interaktiver Kommunikation eine Signatur moderner Gesellschaften. Er geht sogar noch einen Schritt weiter: Gerade die Techniken und Prozesse, die für Anonymisierung und Entbettung der Lebenswelt verantwortlich sind, lassen sich gleichzeitig für die Neuschaffung sozialer Netze und der Pflege intimer Beziehungen in Anspruch nehmen. Paradoxerweise werden heute Globalisierungs- und Entbettungsprozesse zu „Vorbedingungen mancher Formen individueller Selbstverwirklichung ...“ (Giddens 1995: 195). Erst mit der Ausdifferenzierung massenmedialer Kommunikation konnte eine Gesellschaft entstehen, in der nicht mehr jeder jeden kennen muß, in der ein lebensweltlicher Pluralismus reifen konnte, ohne daß die Möglichkeit verloren ging, sich auf eine gemeinsame Realität zu beziehen und persönliche Beziehungen zu entwickeln. Durch die Teilnahme am öffentlichen Geschehen können auch solche Personen Wissen teilen, die sich nicht kennen und nie zu Gesicht bekommen haben. Erst jetzt eröffnen sich Räume für zurückgezogene, anonymisierte Lebensformen, da auch so der (durch jene reflexive Erwartungsstruktur der Massenkommunikation erzeugte) Kontakt zur (massenmedial aufbereiteten) Realität und damit zu anderen Personen gewährleistet bleibt. Gleichzeitig wird durch die medientechnisch erzeugte Öffentlichkeit sowohl für die Alltagskommunikation als auch auf der Ebene gesellschaftlicher Teilssysteme ein Raum für gesteigerte Selbstbeobachtung und -artikulation geschaffen. Dies zeigt bereits die Entwicklung der Printmedien: Das Lesen von Texten fasziniert, nicht nur weil es die Erfahrung einer vom eigenen Standpunkt abweichenden Deutung der Welt ermöglicht, sondern weil es zu beobachten erlaubt, wie andere, dem Leser fremde Personen die beschriebene Welt beobachten und erfahren. Jeder Leser oder Zuschauer hat die Möglichkeit, sein Textverständnis mit dem eines anderen $\mathrm{zu}$ vergleichen und dadurch zu profilieren. ${ }^{16} \mathrm{Hinzu}$

\footnotetext{
${ }^{16}$ Vergleichbares gilt für die audiovisuellen Medien. So beschreibt etwa Meyrowitz (1990: 254) das Fernsehen als einen gewaltigen Spiegel, in dem nicht nur Gemeinsamkeiten, sondern auch Unterschiede in den Lebensformen erkannt werden können. Das bis auf den heutigen Tag feststellbare Bemühen des Fernsehens, einen Zuschauer anzusprechen, der, wie Hickethier (1994:271) ausführt, eine „moderate Mitte zwischen Anpassung und Beharrung im Modernisierungsstrom" verkörpert, bringt dem realen Zuschauer die Besonderheiten seines eigenen Lebensstils zu Bewußtsein.
}

kommt, daß im Gegensatz zur Interaktion, die eher entmutigt, einen abweichende Standpunkt einzunehmen, die meist einsame Lektüre, in der der Leser von der Verpflichtung zu (inter)agieren befreit wird, zu kritischer Distanznahme und Reflexion auffordert. ${ }^{17}$ Obwohl es sich also bei der Lektüre um eine einseitige, also interaktionfreie Kommunikation handelt, steht fest, daB durch Schreiben bzw. Lesen der Grad an Individualität und persönlicher Erfahrung zugenommen hat. Die Entstehung von Persönlichkeit und Individualität ist eng verknüpft mit der Verbreitung von Printmedien und Schriftkultur (vgl. McLuhan $1968 \mathrm{ff}$.; Goody/Watt 1981: 114 ff.).

Die Unterbrechung der Interaktion und Rollenverteilung zwischen Sender und Empfänger erzeugt also keinen normativen Anpassungszwang an publizierte Informationen und Meinungen, wie vor allem die kritische Medientheorie befürchtete. Dieser wäre freilich auch mit der Ausdifferenzierung und operativen Geschlossenheit der Teilsysteme moderner Gesellschaften gar nicht zu vereinbaren. ${ }^{18}$ "Ein-Weg-Kommunikation" ist eine wichtige Voraussetzung für die Ausbildung höherer kommunikativer Freiheitsgrade in der Behandlung relevanter gesellschaftlicher Ereignisse - und zwar, wie zuletzt Luhmann (1996) ausführt, sowohl

17 Nichtumsonst fuhlten sich autorităre Gesellschaften, wie die des 17 . und 18. Jahrhunderts, durch ein sich selbst erlebendes Subjekt - ob nun als literarische Vorlage, wie in der Figur des jungen Werther, oder real existierend herausgefordert, weil es sich nicht mehr ausschließlich an äußeren Maßstäben gebunden fühlt, sondern auch an inneren Werten zu orientieren vermag und damit für die Obrigkeit unkalkulierbar wird. Warnungen vor allzu phantasiefördernder und emotionalisierender Literatur hatten deshalb weniger das Wohl des Lesers als das der gesellschaftlichen Autoritäten im Auge (vgl. Bachmair 1996: 306).

${ }^{18}$ Luhmann $(1990$; 1992) gebraucht in diesem Zusammenhang die instruktive Metapher des Spiegels, um die Relevanz der Brennpunkte öfentlicher Aufmerksamkeit für die Profilierung politischer Standpunkte zu verdeutlichen. Die öffentliche Meinung dient der Politik nicht nur als Sensor für die Interessen und Bedürfnisse der Bürger. Sie erlaubt ferner jedem politischen Akteur sich selbst und die anderen Akteure des politischen Systems zu sehen, insofern in den Medien darüber berichtet wird, wie sie sich gegenüber einem Thema der öffentlichen Meinung verhalten. Auf diese Weise lassen sich nicht nur Unterschiede in den politischen Positionen und Strategien feststellen. Veröffentlichte Stellungnahmen und Entscheidungen anderer Akteure bieten daruberhinaus Orientierungspunkte für die Entwicklung und Profilierung eigener Präferenzen und Strategien. 
für den Sender, der nun nur noch nach Maßgabe interner, nicht-verallgemeinerungsfähiger Kriterien darüber entscheidet, worüber er wie berichtet, als auch für den Rezipienten, der aufgrund der strukturell garantierten Widerspruchsmöglichkeit die Themen der Medien in Kommunikation mit anderen zum Gegenstand persönlicher Meinungsbildung machen kann. ${ }^{19}$ Die Anonymität des Mediums, also der Verzicht, das Geschriebene oder Gesendete an einen besonderen Rezipienten zu adressieren, ebenso wie die distanzierte Beobachterrolle machen deshalb Sinn. Der Rezipient gewinnt gerade hierdurch Reflexionspotentiale und größere Freiheitsgrade in der Interpretation der Medienangebote. Wäre beispielsweise die Rezeption eines Textes oder einer Sendung nicht interaktionsfrei und der Leser als Entscheider in den Verlauf einer textbasierten Argumentation oder filmischen Handlung involviert, so befände er sich nun zwar in der - von vielen Medientheoretikern geforderten - Mitspielerrolle, allerdings verlöre er nun die Möglichkeit, sich von der Andersartigkeit und Differenz fremder Perspektiven und Gedankengänge irritieren zu lassen. Interaktionsgewinne wären mit Reflexionsverlusten zu bezahlen. Wird hingegen auf Interaktion verzichtet und ein identischer, von den Reaktionen der Empfänger unabhängiger (filmischer oder literarischer) Text an ein anonymes Publikum gerichtet, werden Möglichkeiten der Fremd- und Selbstthematisierung erschlossen, die einer ausschließlich auf interaktive Kommunikation angewiesenen Gesellschaft unbekannt sind.

\section{Medien zweiter Ordnung}

Die zivilisationskritischen Klage über die fehlende bzw. auf einzelne Sinne reduzierte Interaktivität technischer Medien reicht zurück bis in die Anfänge der Schriftlichkeit. Kritik an Schriftkultur setzt sich bis in die Spätromantik fort, in der die Schrift als Entfremdung von einem Naturzustand begriffen wird, in dem einzig die mündliche Rede beheimatet ist (vgl. Goody und Watt 1981: $63 \mathrm{ff}$.). Auch heute noch werden (vor allem die audiovisuellen) Mediatisierungen dieses Ursprungsmodells menschlicher Kommunikation als Zerfallsprozesse einer allein im verständigungsorientierten Handeln sich gründenden Öffentlichkeit gewertet.

19 Auch die öffentlichen Meinung kann die Politik lediglich auffordern, ein Thema zur Kenntnis zu nehmen. Sie kann Kommunikationsprozesse anstoßen, nicht jedoch deren Verlauf bestimmen.
Diese Einstellung zu den Medien wird seit einigen Jahren durch die paradox anmutende Vorstellung irritiert, Dialog und Interaktion ließen sich in technischen Medien wiederherstellen.

Demgegenuber wird hier die These vertreten, daB gerade die Dialog-Metaphorik vieler Analysen und Kommentare zu einer völligen Fehleinschätzung der tatsächlichen kommunikativen Potentiale der neuen Medien verleitet, da gerade sie die gegenwärtig fortgeschrittenste Form einer Auflösung des strukturellen Kontexts interaktiver Kommunikation darstellen. Kommunikation unter Anwesenden bildet einen situativen Zusammenhang zwischen Mitteilung und Verstehen. Die Interaktion ist hier eingebettet in einen gemeinsamen Wahrnehmungs- und Handlungsraum. Selbst Massenkommunikation, in der dieser Zusammenhang bereits brüchig wird, da Mitteilung und Verstehen einer Information räumlich und zeitlich entkoppelt werden, vermittelt ihren Teilnehmern die Möglichkeit einer Bezugnahme auf eine gemeinsame Realität. Obwohl Kommunikatoren und Rezipienten sich i.d.R. nicht kennen und diese Anonymität offen läßt, wie der Rezipient reagieren wird, bleibt doch der Inhalt der Mitteilung für beide Seiten identisch.

Dies ändert sich, sobald Computer die Kommunikation zwischen Sender und Empfänger vermitteln. Folgt man einem Theorievorschlag von Esposito (1993), dann liegt das Besondere interaktiver Medien in der Kontingenzsteigerung der Kommunikation. Computermedien enthalten zwar Programme, die ihre internen Verarbeitungsmechanismen determinieren und insofern das, was Computer "tun", berechenbar machen. Gleichwohl ist das, was sie schlieBlich als output erzeugen, für die Nutzer selbst unberechenbar und damit überraschend. Daten werden jetzt nicht mehr nur aufgenommen und verbreitet, wie bei den Printmedien, sondern außerdem - in Abhängigkeit von den jeweils installierten Programmen und den Operationen des Nutzers - verändert. Projekte einer Interaktivierung der Medien, wie etwa die „elektronische Zeitung“ der Firma Pointcast, lassen sich deshalb vor dem Hintergrund der voranstehenden Überlegungen zur Funktion der Massenmedien als eine radikale Entkopplung sämtlicher Kommunikationskomponenten begreifen. Mitteilung und Verstehen werden jetzt nicht nur unter zeitlichen und räumlichen, sondern auch unter sachlichen Gesichtspunkten auseinandergerissen (vgl. Ellrich 1996). Das Eingeben und Abrufen von Daten werden durch das Dazwischentreten von Programmen, die diese Daten speichern und aufbereiten, 
zu selbstständigen isolierten Prozessen. Unterbrochen wird damit der Kontakt nicht nur zwischen Sender und Empfänger, sondern auch zwischen den Empfängern. Die für Massenkommunikation typische „Reflexivität des Wissens“" (Merten 1977) löst sich hier wieder auf, wie sich am fiktiven Beispiel der Lektüre einer elektronischen Zeitung zeigen läßt: Der Leser weiß jetzt nicht nur nicht, was der Anbieter der Zeitung weiß bzw. welche Informationen dieser in das System eingegeben hat oder eingeben wird. Er weiß auch nicht, welche Informationen andere Leser wann und wo dem Medium entnehmen werden. Umgekehrt gilt dasselbe für den Anbieter. Obwohl er die Daten eingibt, weiß er weder, wie und wann sich jemand damit befassen wird, noch, welche Daten von den Leser entnommen werden. Die Daten sind bereits hier, obwohl es sich um ein Medium handelt, bei dem zwischen Sender und Rezipient noch unterschieden werden kann, nicht mehr fixiert, wie noch beim herkömmlichen Buch oder einer gewöhnlichen Zeitung, sondern werden immer mehr zu einer Funktion von Nutzerentscheidungen. Die Information ist damit weder in der Beziehung zwischen Sender und Empfänger noch für die Rezipienten als Publikum identisch.

Man kann diesen Vorgang als Befreiung des Nutzers von vorgefertigten Angeboten und themenbezogenen Filtertechniken der Massenmedien begreifen und die weitere Medienentwicklung als Aufhebung einer strikten Rollentrennung zwischen Sender und Empfänger beschreiben. Vor dem Hintergrund der bisherigen Argumentation ist die eigentliche Herausforderung medialer Interaktion aber wohl in dem Fehlen einer gemeinsamen thematischen Referenz und Bündelung gesellschaftlicher Aufmerksamkeit zu sehen. Welche Konsequenzen sich aus dieser weiteren Kontingenzsteigerung für Kommunikation und Medienumgang ergeben, ist derzeit noch völlig ungewiß. Allerdings weckt die hier skizzierte Problemformel Bedenken sowohl hinsichtlich der Vision eines künstlichen Dialogs zwischen nichtanwesenden Personen als auch gegenüber der Erwartung einer netzwerkbasierten Öffentlichkeit:

(1) Deutlich wird nun, daß die computervermittelten Möglichkeiten „to talk back“ (Rogers 1986: 34) nicht mit interpersonaler Kommunikation verwechselt werden dürfen. Die für interaktive Kommunikation konstitutive Reflexivität der Wahrnehmungen wird hier verhindert durch zwischengeschaltete Programme, die Mitteilung und Verstehen zu unabhängigen Prozessen machen. Es fehlt deshalb nicht nur ein von den Teilnehmern des Kommunikationssystems geteilter Wahrnehmungs- und Handlungsraum, sondern auch die für interaktive Kommunikation typische Kontrollmöglichkeit, das Gesagte zu hören und anhand nonverbaler Zeichen erahnen zu können, ob jemand auch wirklich meint, was er sagt. Nicht zuletzt dieses Fehlen nonverbaler Orientierungen und Kontrollmöglichkeiten sowie die Möglichkeit, nach Belieben in die Kommunikation ein- und wieder auszusteigen, hat in den Computernetzen zu einem ungehemmten Kommunikationsverhalten geführt (vgl. Höflich 1996: 84 ff.). Auch wenn durch die Einführung parasprachlicher Codes (z. B. durch sog. Emoticons) und Regelungen im Sinne einer Mediengrammatik dieses Verhalten allmählich gebändigt wird, kann dies doch nicht darüber hinwegtäuschen, daB der Reiz computervermittelter Kommunikation gerade nicht in der sozialen Nähe und persönlichen Begegnung, sondern in der "Maskerade und Anonymität" ihrer Teilnehmer (Rötzer 1996: 119) liegt. Elektronische Gemeinschaften sind Gemeinschaften ohne Verpflichtung. Kommunikation mit dem Computer läßt sich deshalb zwar durchaus als interaktiv beschreiben, ohne daß hierbei jedoch davon ausgegangen werden $\mathrm{muB}, \mathrm{da} B$ eine Angleichung an natürliche Interaktionen bzw. Dialoge stattfindet. Wie Esposito (1993) ausführt, steht bei der computervermittelten Kommunikation die Interaktion mit einer Maschine im Vordergrund, die auch dann ihren Dienst tut, wenn mehrere Teilnehmer gleichzeitig mit ihr interagieren und dabei den Eindruck gewinnen, sie kommunizierten miteinander. Durch die maschinelle Vermittlung bleibt die Interaktion anonym (vgl. Myers 1987). Ob jemand mit einem Menschen vermittels der Maschine oder doch nur mit einer Maschine kommuniziert habe, bleibt für ihn als Nutzer unerfindlich. Allerdings hat das (Nicht-)Wissen um diese Differenz für das Gelingen der Kommunikation auch keinerlei Bedeutung.

(2) Ebensowenig lassen sich Funktionen der Massenmedien auf interaktive Medien übertragen. Die Möglichkeit - man könnte auch sagen: der Zwang - zur individuellen Einmischung in das mediale Geschehen verhindert, daß sich der für Massenkommunikation typische Weltbezug einstellen kann. Wenn jeder aus einer unbegrenzten Menge von Bildern, Tönen und Texten sein persönliches Medienmenü zusammenstellen muß, die Selektionslast folglich nicht mehr auf der Sender-, sondern auf der Empfängerseite liegt, kann nicht mehr erwartet werden, daß sich gleichzeitig alle Teilnehmer mit demselben Thema befassen. Der 
Sinn interaktiver Medien liegt nicht darin, daß gleiche Informationen gleichzeitig alle Rezipienten erreichen, sondern in den Möglichkeiten, die Relationen zwischen Informationen, dem Zeitpunkt ihrer Weitergabe und Rezeption und der Menge ihrer Rezipienten zu variieren. Interaktive Medien gründen deshalb - entgegen den Annahmen der politisierten Fraktion ihrer Befürworter kein Gemeinwesen, sondern eher eine „ZweierBeziehung". Ihre Nutzung gleicht mehr dem Telefonieren als dem Fernsehen oder Zeitunglesen: „Die Wahrnehmung aus der Perspektive der ersten Person, die im Cyberspace möglich ist, oder die individuelle Entscheidung für bestimmte Pfade etwa in einem Hypertext lassen sich", wie Rötzer (1996: 129) schreibt, „nicht verallgemeinern ..." Interaktive Medien verhindern die Unterstellung eines gemeinsamen Themas bzw. eine „WirPerspektive", wie sie sich in der reflexiven Erwartungsstruktur der Massenkommunikation einstellt. Je fortgeschrittener die Möglichkeiten einer individuellen Einmischung in das mediale Geschehen sein werden und je mehr Such-und Erreichbarkeitsmanagement-Systeme die Angebote in den Netzen auf persönliche Interessen durchforsten und filtern, desto unwahrscheinlicher wird, daB zwei Rezipienten dasselbe sehen. Zwar kann jeder -vorausgesetzt, er wird durch keine Zugangskontrollen daran gehindert - alles sehen und lesen, aber jeder sieht oder liest etwas anderes. Interaktive Medien können deshalb nicht wie Massenmedien funktionieren, selbst wenn sie massenhaft genutzt würden. Mit der Möglichkeit für den Nutzer zu kommunizieren werden nicht nur etablierte Rollenverteilungen (z. B. Journalisten vs. Leser/Zuschauer) und darauf aufbauende Vertrauensbeziehungen außer Kraft gesetzt. Ebenso erlischt der Anspruch, etwas Allgemeines mit der Botschaft zu verbinden. Botschaften im Netz sind zwangsläufig als Mitteilung aufzufassen und somit partikular und zurechenbar auf konkrete Mitteilungsabsichten. Sie sind ebenso zwangsläufig immer nur für spezielle Interessengruppen und Nutzerkollektive interessant, jedoch nicht im Sinne verallgemeinerungsfähiger Themen öffentlich. Jedenfalls wird durch die unbegrenzte Anbietervielfalt und die Möglichkeit persönlicher Eingriffe in mediale Abläufe die für das System der Öffentlichkeit konstituitive kontextneutralisierende Funktion abstrakter und anonymer Aufmerksamkeitsregeln unterlaufen und damit das Medium allenfalls für Spezialkulturen, die konkretere Selelektionsregeln zu teilen bereit sind, integrationsfähig.
(3) Was sich in diesem gegenüber dem vertrauten Medienumgang veränderten Arrangement der Beziehungen zwischen Sender und Empfänger ebenfalls abzuzeichnen beginnt, ist, da $\beta$ sich die Vorstellung von Wirklichkeit und damit auch die Einstellung zu den Medien generell verändert (vgl. Schmidt 1994: 276 ff.). Interaktive Medien, so Spangenberg (1996), kommunizieren ihre Konstruktivität. Sie denunzieren das für die ,alten Medien" noch gültige Kriterium der Realitätsangemessenheit und markieren - überspitzt formuliert - den Wandel von einem „ontologischen“ zu einem „konstruktivistischen“ Verständnis medial inszenierter Wirklichkeit. Denn wird der Interaktionsverzicht zwischen Sender und Empfänger aufgehoben, entfällt gleichzeitig der Latenzschutz für die Konstruktionsabhängigkeit der Medienrealität. Nicht mehr die durch das System der Massenkommunikation erzeugte 'objektive' Welt, sondern die durch das neue Medium ermöglichte Vielfalt von Wirklichkeitsdarstellungen erregt nun die Aufmerksamkeit der Teilnehmer. ${ }^{20}$ Das Medium verliert seine „Unsichtbarkeit“: Esposito (1993) unterscheidet hier zwischen "Medium", das als Vermittelndes nicht in Erscheinung tritt, und „Maschine", die sich gerade dadurch auszeichnet, daß sie Eingaben des Nutzers verändert und mit unerwarteten Reaktionen überrascht. In Anlehnung an Schmidt und Luhmann ließen sich interaktive Medien deshalb auch als „Medien zweiter Ordnung“ beschreiben: Die Aussagen dieses Medientyps lassen sich nicht wie (künstlich erzeugte) objektive Sachverhalte behandeln, sondern werden auf die Entscheidungen eines (maschinellen) Kommunikationsteilnehmers zurechenbar. Das Vermittelnde, die Tatsache, daß Sender/Empfänger und Empfänger/Sender kommunizieren, läßt sich jetzt nicht länger ignorieren, ja, macht den eigentlichen Reiz der neuen Medien aus. Die Nutzer interaktiver Medien bewegen sich in symbolischen Welten, in denen sie ohne den Rückhalt einer (fiktiv unterstellten) objektiven Außenwelt und auch ohne die

20 Hier geht es zunăchst nur um das Verhältnis zur Wirklichkeit im Rahmen der Mediennutzung. Nur in dieser Hinsicht wird behauptet, daß sich nicht mehr länger von ontologischen Gewißheiten ausgehen läßt und stattdessen das Vermittelnde in den Vordergrund rückt. Inwieweit davon auch die außermediale Einstellung zur Wirklichkeit betroffen ist, wird hier offen gelassen. $\mathrm{Zu}$ vermuten ist jedenfalls nicht ein "Verschwinden der Wirklichkeit", wie schon dem Fernsehen vorgeworfen wurde, eher der Effekt einer gesteigerten Sensibilităt für die generelle Unterscheidungsabhängigkeit von Wirklichkeitsvorstellungen (vgl. Schmidt 1994:284). 
Sicherheit einer von allen Teilnehmern geteilten Sicht der Welt auszukommen haben. Auch die bislang unsichtbaren „constraints" hinter jeder Medienaussage, also jene fraglos hingenommenen und unbeobachteten Codierungspraktiken, wie sie in den Massenmedien die Komplexität der Welt auf ein beobachtbares $\mathrm{Ma} B$ herabsenken, rücken im Kontext medialer Interaktion in den Vordergrund und werden zwangsläufig als eine aktualisierte von vielen anderen möglichen Formen der Auswahl- und Präsentation der Daten wahrgenommen. Interaktive Medien läuten ein Stadium reflexiver Mediennutzung ein, ste schärfen den „Möglichkeitssinn“ ihres Nutzers und prägen sein "mediales Wirklichkeitsbewußtsein“ (vgl. Wagner 1993).

Diese Entwicklung bekommen paradoxerweise vor allem jene Nutzer zu spüren, die in den neuen Kommunikationsnetzen ein ideales Verbreitungsmedium für Ideen und Erfahrungen sehen, die in den herkömmlichen Verbreitungsmedien keine Berücksichtigung finden. So berichtet Freyermuth (1996) von sog. "Verschwörungstheoretikern“, die zu Tausenden ihr Wissen über die „wahren" Hintergründe der täglichen Katastrophen und Krisen im Netz verbreiten und dort ein dankbares Publikum für ihre teilweise geradezu aberwitzigen und paranoiden Vorstellungen finden. Ihre Botschaft ist die Differenz zwischen der „Scheinwirklichkeit", wie sie vor allem durch die Massenpresse erzeugt wird, und der „realen Wirklichkeit", die sich hinter offiziellen Darstellungen und Verlautbarungen verbirgt. Der Sinn ihrer Frage: „Was steckt dahinter?" scheint jedoch im neuen Medium immer weniger zu überzeugen: „Die Leichtigkeit, mit der sich Digitalisiertes verändern läßt, fordert gerade$\mathrm{zu}$ auf, Worte und Bilder nach den eigenen Bedürfnissen umzumodeln. Unter solchen Umständen verweisen die Materialien (der Verschwörungsfanatiker, J.W.) zuverlässig auf nichts Drittes mehr und können als 'Beweis' allein für die eigenen Absichten dienen. ... Qua Medium ironisiert daher der Cyberspace die verbissene Bastelei von MonoWeltbildern und verzaubert, was in der analogen Realität Ernst war, in ein Computerspiel, einen digitalen Cyberspaß, bei dem multiple Realitäten und viele Wahrheiten koexistieren" (Freyermuth 1996: 176).

Beschränkte sich unter massenkommunikativen Bedingungen das Beobachten anderer Beobachtungen noch auf den Empfängerkreis, so richtet es sich nun auf den Sender bzw. dessen Selektionen. Es gibt deshalb in einem solchen System zunächst - prinzipiell - nichts, was nicht kommunizierbar wäre, da es keine unbefragten Aufmerksamkeitsregeln mehr gibt. ${ }^{21}$ Anders als in den Massenmedien, wo der Aufmerksamkeitswert einer Meldung sich allein aus ihrer Veröffentlichung ergibt, folgt hier die Aufmerksamkeit - da grundsätzlich keine Daten ausgeschlossen werden - den Unterschieden in den Darstellungsverfahren. Wird der Referenzstatus einer Information immer prekärer und kann sich der Empfänger immer weniger sicher sein, ob sich „hinter" der medialen Realität überhaupt noch eine exmediale Bezugwirklichkeit befindet, droht ein Umschlagen dieser Unsicherheit in Gleichgültigkeit gegenüber Fragen der Wirklichkeitsangemessenheit medial verfaßter Aussagen. ${ }^{22}$ Immer wichtiger wird, ob eine Aussage den Geschmack, das ästhetische Empfinden der Rezipienten trifft. Der Wert einer Aussage bemißt sich nun stärker an dem subjektiven Erleben ihrer Empfänger (vgl. Krotz 1995a: 459). Dies erklärt möglicherweise den hohen Stellenwert, den Praktiken einer Visualisierung bzw. Ästhetisierung der Mitteilung in diversen Informations- und Kommunikationsdiensten des Internet einnehmen (vgl Becker 1996). Nachdem über Jahrhunderte hinweg von der Einführung der Schrift bis hin zu den elektronischen Massenmedien der Informationsaspekt im Vordergrund stand, wird nun mit den neuen Medien die Unterscheidungsqualität der Kommunikation zum vorrangigen Attraktor von Aufmerksamkeit (vgl. Spangenberg 1991). Demnach ist im Kontext der neuen Medien auch weniger die Auswahl von Themen, sondern vor allem

21 Jeder Versuch, Regeln zu installieren und verbindlich zu machen, setzt sich unweigerlich der Beobachtung und Kritik aus. Computernetze erzeugen den Eindruck eines unberechenbaren und "flilchtigen" Mediums, in dem Strukturen oft nur von sehr kurzer Dauer sind. Sie gleichen Experimentierfeldern, auf denen nicht nur ständig nach neuen Formen der Kommunikation, sondern auch der Auswahl- und Präsentiertechniken gesucht wird. Diese Problematik gewinnt proportional zur beanspruchten Reichweite der Regeln an Schärfe. Die von vielen befürchtete oder herbeigesehnte internationalisierte und globalisierte Netzkommunikation mit einer uniformen Sprachregelung und weltweit verbreiteten gleichartigen Kommunikationstechniken ist u.a. auch deshalb (vorerst) Vision geblieben, weil sich damit ein gewaltiger, z.Z. nicht lösbarer Regelungs- und Kontrollaufwand verbindet (vgl. Kubicek 1995; Weingarten 1996).

22 So berichtet Schmidt (1994:279) von Untersuchungen uber Jugendliche, die mit den neuen elektronischen Medien groß geworden sind und dazu tendieren, nicht mehr nach empirischen Referenzen von Medienaussagen zu suchen, sondern sich stattdessen darauf beschränken, jedem Medium seine eigene Wirklichkeit zuzuordnen. 
die Beschaffenheit der Auswahl- und Präsentationsverfahren für die Annahme von Medienangeboten ausschlaggebend. Entscheidend für die Akzeptanz eines Mediums wird dessen „Materialität", das heißt seine Ästhetik, seine Auswahlverfahren, sein Bezug zu anderen Medien und natürlich auch seine Interaktivität (vgl. Schmidt 1995; Welsch 1995).

Unterstützt werden diese Vermutungen durch empirische Untersuchungen über Benutzer von Datennetzen (vgl. Wetzstein u.a. 1995; Jones 1995; Höflich 1996; Hoffmann 1996; Kubicek/Schmid 1996). Sie zeigen, wie mediale Interaktionen die Bildung sog. elektronischer Gemeinschaften fördern, deren Zusammenhalt auf spezifischen, vor allem von expressiven Stilelementen geprägten Aneignungs- und Gebrauchsformen beruht. So hat sich beispielsweise um die verschiedenen Kommunikationsdienste im Internet eine Vielfalt von Nutzungskulturen und Kommunikationsstilen herausgebildet. Auffällig sind vor allem die Formen der symbolischen Abgrenzung nach außen und nach innen sowie die spezifischen Verhaltensstandards und Rituale, die den jeweiligen Kulturen eine Identität verleihen. Daß Sich-Mitteilen steht hier im Vordergrund, während die Information geringere Bedeutung hat. ${ }^{23}$ Zumindest in der gegenwärtigen Nutzungsphase geht es im Internet scheinbar weniger darum, den referentiellen Rahmen, also die thematischen Beschränkungen massenmedialer Berichterstattung zu überwinden, als vielmehr um das Experimentieren mit ungewöhnlichen Formen der Selbstinszenierung und um die Erfahrung neuartiger Kommunikations-und Erlebnisqualitäten (vgl. Hoffmann 1996; Vogelsang 1996). Der für die Massenkommunikation charakteristische Weltbezug tritt hier zurück hinter das Ausnutzen der ungewöhnlichen Formbarkeit des neuen Mediums, das Experimentieren mit medialen Inszenie-

${ }^{23}$ Interessant ist hier der Hinweis von Müller (1996: 152) auf Forschungen uber das Teilnehmerverhalten in USamerikanischen „Call in Talk Radio"-Sendungen, die die Möglichkeit einer telefonischen Kontaktaufnahme der Zuhörer mit einem Moderator bieten, der u.U. gleichzeitig im Fernsehen zu sehen ist. Festgestellt wurde nicht nur, daß ein verschwindend geringer Prozentsatz der Rezipienten zum Telefon greift, sondern daß der große Rest die Rolle des unbeteiligten Zuhörers/Zuschauers genieBt, der zum Zeugen teilweiser dramatischer Selbstinszenierung wird. D.h., interaktive Sendungen verdanken ihre Attraktivităt der Tatsache, daB sie immer auch traditionelle Unterhaltung darstellen, so wie für den (Großteil der) Anrufer ("Interakteur") das (heimliche) Teilnahmemotiv darin besteht, sich vor einem Publikum darstellen zu können. rungsritualen und Spielen mit brüchig gewordenen Unterscheidungen wie Subjekt und Objekt, Sein und Schein, Realität und Fiktion (vgl. Schmidt 1995). Die Interaktivität der Medien unterstützt scheinbar Prozesse der Selbsterfahrung und Grüdung von Sonderkulturen - jedenfalls nicht die Erzeugung eines anonymen Publikums.

\section{Fazit}

Die hier zusammengetragenen Überlegungen sollten deutlich machen, da $B$ in der Medienevolution weder der Trend zu einer hoffnungslosen Vereinzelung, noch der einer gesichtslosen „Massifizierung " (Flusser) der Menschen erkennbar sind. Das Gegenteil ist der Fall. Presse und Hörfunk/Fernsehen übernehmen für die Gesellschaft die paradoxe Aufgabe, durch die Bereitstellung Aufmerksamkeit fokussierender Beschreibungen der Welt gleichzeitig Spielräume für die Individualisierung und Anonymisierung ihrer Rezipienten zu eröffnen. Da hierfür die technische Unterbrechung zwischen Sender und Empfänger unabdinglich ist, erweisen sich Bemühungen um eine Re-Dialogisierung der Massenmedien oder die Erwartung, Funktionen der Massenmedien auf interaktive Medien übertragen zu können, als eigentümlich fehlgeleitet. Mit ihren Prognosen und Erwartungen stellen die Protagonisten einer neuen interaktiven Medienwelt kommunikative Funktionen eines Medientyps in Frage, ohne daß sie in der Lage wären, funktionale Äquivalente anzubieten. Die gegenwärtig zu beobachtende Zurückhaltung in der Nutzung interaktiver Medien ließe sich deshalb auch als Weigerung verstehen, auf Gratifikationen der Massenkommunikation zu verzichten (vgl. Vorderer 1995). ${ }^{24}$

Vorstellungen wie die einer 'persönlichen Zeitung' oder die bereits in den 70er Jahren verbreitete $\mathrm{Vi}$ sion, in Zukunft könne jeder über sein eigenes Massenmedium verfügen, führen deshalb ins $\mathrm{Ab}$ surde. Es uberrascht nicht, wenn Versuche scheitern, die aus Fernsehen, Kino oder Zeitungen „Zwei-Wege-Medien“ machen wollen. Sie verken-

${ }^{24}$ Möglicherweise liegt darin auch eine Erklärung für die „Wiederentdeckung“ des Kinos in den letzten Jahren. Kino ermöglicht die gemeinsame Teilhabe an einem nicht beeinfluBbaren Ereignis, wăhrend Fernsehen aufgrund der Monotonie seiner Kanäle, der man sich nur noch durch Zapping erwehren kann, zum Individualmedium mutiert. Kino bietet folglich einen thematischen Bezugsrahmen für anschließende Diskussionen, die durch Fernsehen eher verhindert werden. 
nen die Crux der Massenmedien, nämlich einerseits mit Hilfe anonymer, an keinen besonderen Zuhörer oder Zuschauer adressierter Kommunikationsangebote Gemeinsamkeit zu erzeugen, andererseits, indem die Zuschauer oder Leser mit Bezug auf einen identischen Hintergrund ihre $\mathrm{Be}$ obachtungen vergleichen, Unterschiede und $\mathbf{A b}$ weichungen zu ermöglichen. Was die Aussagen und Bilder dieser Medien interessant und (beispielsweise für die Politik) entscheidungsrelevant macht, ist, daß sie als Informationen einem unbegrenzten Publikum präsentiert werden, und erst in zweiter Linie, daß sie als Ereignisse tatsächlich vorgekommen sind.

Andererseits kann das Vordringen der neuen Medien mit ihren Angeboten der Individualkommunikation nicht übersehen werden. Es läßt sich deshalb immer weniger von den Medien sprechen. Statt von einer „Hybridisierung“ der Medien oder von einer Ab-oder Auflösung der Massenkommunikation zu sprechen, wäre es jedoch sinnvoller, die strukturelle Andersartigkeit interaktiver Medien gegenüber den vertrauten Medien zu berücksichtigen und von einer weiteren Differenzierung des Mediensystems auszugehen. Nicht die Verdrängung oder Ablösung alter durch neue Medien, sondern die Neuformatierung eines erweiterten Mediensystem ist zu erwarten. Da sich gegenwärtig bei den neuen Medien noch vieles ,im Fluß" befindet, bleibt zukünftigen Forschungen vorbehalten, diesen Prozeß genauer zu analysieren und zu kommentieren.

\section{Literatur}

Adorno, T. W., 1963: Neun kritische Modelle. Frankfurt/M. Baake, D., (Hrsg.), 1974: Kritische Medientheorien. Konzepte und Kommentare. Munchen

Bachmair, B., 1996: Fernsehkultur. Subjektivität in einer Welt bewegter Bilder. Opladen

Becker, B., 1996: Ästhetisierung der Medien. (unveröff. Ms.)

Böckelmann, F., 1975: Theorie der Massenkommunikation. Frankfurt/M.

Bollman, S. (Hrsg.), 1995: Kursbuch Neue Medien, Trend in Wirtschaft und Politik, Wissenschaft und Kultur. Mannheim

Bollmann, S./Heibach, C. (Hrsg.), 1996: Kursbuch Internet. Mannheim

Bolz, N., 1993: Vom Ende der Gutenberg-Galaxis. Mannheim

Brecht, B., 1967: Radiotheorie. S. 114-134 in: Gesammelte Werke, Band 18, Schriften zur Literatur und Kunst. Frankfurt/M.
Bretz, R., 1983: Media for Interactive Communication. Beverly Hills, London, New Delhi

Buchstein, H., 1996: Cyberbürger und Demokratietheorie. Deutsche Zeitschrift für Philosophie 44: 583-607

Charlton, M., 1993: Methoden der Erforschung von Medienaneignungsprozessen. S.11-26 in: Holly, W./PUschel, U. (Hrsg.), Medienrezeption als Aneignung. Methoden und Perspektiven qualitativer Medienforschung. Opladen

Dervin, B., 1983: Information as a User Construct: The Relevance of Perceived Information Needs to Synthesis and Interpretation. S. 153-184 in: Ward, S.A./Reed, L.J. (Hrsg.), Knowledge Structures and Use: Implications for Synthesis and Interpretation. Philadelphia

Dutton, W. H., 1994: Driving into the Future of Communications? Check the Rear View Mirror. (unveröff. Ms.)

Ellrich, L., 1996: Sein und Schein. Wie postmodern ist das systemtheoretische Konzept der elektronischen Medien? Deutsche Zeitschrift fur Soziologie 44: 559-582

Enzensberger, H. M., 1970: Baukasten zu einer Theorie der Medien. S. 160-173 in: Kursbuch 20

Esposito, E., 1993: Der Computer als Medium und Maschine. Zeitschrift für Soziologie 22: 338-354

Fabris, H. H., 1985: Der Mythos der Massenkommunikation oder das Dilemma der Kommunikationswissenschaft. Plädoyer für die Entwicklung der Medien- zur allgemeinen Kommunikationswissenschaft. Österreichisches Jahrbuch fur Kommunikationswissenschaft 3: 125-137

Faulstich, W., 1991: Medientheorien: Eine Einfuhrung und Überblick. Göttingen

Faulstich, W. (Hrsg.), 1994: Grundwissen Medien. München

Florian , M., 1996: „Soziomedia“ als Virtualisierung von Kultur? Überlegungen zur Sozialmetaphorik in der KI an der Grenze zwischen Realităt und Virtualität. S.101-148 in: Becker, B./Lischka, C./Wehner, J. (Hrsg.), Kultur - Medien - Kunstliche Intelligenz. Beiträge zum Workshop „Kunstliche Intelligenz - Medien - Kultur während der 19. Jahrestagung für Künstliche Intelligenz (KI-95) 11.-13. September 1995 in Bielefeld, St. Augustin

Flusser, V., 1995: Verbündelung oder Vernetzung? S. 1523 in: Bollman, S. (Hrsg.), Kursbuch Neue Medien. Trend in Wirtschaft und Politik, Wissenschaft und Kultur. Mannheim

Freyermuth, G. S., 1996: Das Internetz der Verschwörer. Eine Reise durch die elektronische Nacht. S. 163-177 in: Bollmann, S./Heibach, C. (Hrsg.), Kursbuch Internet. Anschlusse an Wirtschaft und Politik, Wissenschaft und Kultur. Mannheim

Gerhards, J., 1994: Politische Öfentlichkeit. Ein systemund akteurstheoretischer Bestimmungsversuch. S. 77105 in: Neidhardt, F. (Hrsg.), Offentlichkeit, öffentliche Meinung, soziale Bewegungen. Sonderheft 34 der Kölner Zeitschrift für Soziologie und Sozialpsychologie. Opladen

Giddens, A., 1995: Konsequenzen der Moderne. Frankfurt/M. 
Gieseke, M., 1992: Natüliche und kunstliche Sprachen. Medienrevolutionen und ihre Auswirkungen auf Sprachen und Sprachbegriffe. S.36-72 in: Gieseke, $M$. (Hrsg.), Sinnenwandel, Sprachwandel, Kulturwandel. Studien zur Vorgeschichte der Informationsgesellschaft. Frankfurt/M.

Goertz, L., 1995: Wie interaktiv sind Medien? Auf dem Weg zu einer Defintion von Interaktivităt. Rundfunk und Fernsehen 43: 477-493

Goody, J./Watt, I., 1981: Konsequenzen der Literalităt. S. 63-122 in: Goody, J./Watt, I./Gough, K. (Hrsg.), Entstehung und Folgen der Schriftkultur. Frankfurt/M.

Großklaus, G., 1995: Medien-Zeit Medien-Raum. Zum Wandel der raumzeitlichen Wahrnehmung in der Moderne. Frankfurt/M.

Hagen, M., 1996: A Road to Electronic Democracy? - Politische Theorie, Politik und der Information Superhighway in den USA. S. 63-85 in: Kleinsteuber, H.J. (Hrsg.), Der „Information Superhighway“. Amerikanische Visionen und Erfahrungen. Opladen

Heinze, T., 1990: Medienanalyse. Ansătze zur Kultur- und Gesellschaftskritik. Opladen

Hickethier, K., 1994: Zwischen Einschalten und Ausschalten, Fernsehgeschichte als Geschichte des Zuschauens. S. 237-306 in: Hickethier, K. (Hrsg.), Institution, Technik und Programm. Rahmenaspekte der Programmgeschichte des Fernsehens. München

Hoffmann, U., 1996: „Request for Comments“: Das Internet und seine Gemeinde. S. 104-117 in: Kubicek, H. (Hrsg.), Jahrbuch für Telekommunikation und Gesellschaft. Heidelberg

Höflich, J. R., 1995: Vom dispersen Publikum zu „elektronischen Gemeinschaften ". Plädoyer für einen erweiterten kommunikationswissenschaftlichen Blickwinkel. Rundfunk und Fernsehen 43: 518-537

Höflich, J. R., 1996: Technisch vermittelte interpersonale Kommunikation. Opladen

Holly, W./Püschel, U. (Hrsg.), 1993: Medienrezeption als Aneignung. Methoden und Perspektiven qualitativer Medienforschung. Opladen

Holzer, H., 1973: Massenkommunikation als Kapitalverwertungsproze $B$ und die Rolle des Publikums. S. 148171 in: Holzer, H. (Hrsg.), Kommunikationssoziologie. Reinbek

Hunziker, P., 1988: Medien, Kommunikation und Gesellschaft. Einfuhrung in die Soziologie der Massenkommunikation. Darmstadt

Jăckel, M., 1995: Interaktion. Soziologische Anmerkungen zu einem Begriff. Rundfunk und Fernsehen 43: 463-476

Janshen, D., 1980: Kabelfernsehen für Gropiusstadt?: Konzept für ein variables Telekommunikationssystem (VTS). Frankfurt/M., New York

Jones, S.G., 1995: Cybersociety. Computer-Mediated Communication and Community. Thousand Oaks, London

Joußen, W., 1991: Massen und Kommunikation. Zur soziologischen Kritik der Wirkungsforschung. Weinheim

Krämer, S., 1995: Spielerische Interaktion. Überlegungen zu unserem Umgang mit Instrumenten. S. 225-236 in:
Rötzer, R. (Hrsg.), Schöne neue Welten? Auf dem Weg zu einer neuen Spielkultur. München

Krol, E., 1995: Die Welt des Internet. Handbuch und Übersicht. Bonn

Krotz, F., 1991: Lebensstile, Lebenswelten und Medien: Zur Theorie und Empirie individuenbezogener Forschungsansätze des Mediengebrauchs. Rundfunk und Fernsehen 39: 317-342

Krotz, F., 1995: Fernsehrezeption kultursoziologisch betrachtet. Der Beitrag der cultural studies zur Konzeption und Erforschung des Mediengebrauchs. Soziale Welt 46: 243-245

Krotz, F., 1995a: Elektronisch mediatisierte Kommunikation. Überlegungen zur Konzeption einiger zuküftiger Forschungsfelder der Kommunikationswissenschaft. Rundfunk und Fernsehen 43: 445-462

Kubicek, H., 1995: Computernetze und die bürgerlichen Freiheitsrechte. Spektrum der Wissenschaft. Dossier 1: Datenautobahnen

Kubicek, H./Seeger, P., 1994: Neue elektronische IuK-Systeme und Alltagsorientierung in der Stadt - Rekonstruktion und Re-Interpretation von Konzepten und Modellversuchen der letzten 20 Jahre. S. 155-180 in: Verbund Sozialwissenschaftliche Technikforschung, Mitteilungen 10, Köln

Kubicek, H./Schmid, U., 1996: Alltagsorientierte Informationssysteme als Medieninnovation. Konzeptionelle Überlegungen zur Erklärung der Schwierigkeiten, „Neue Medien“ und „Multimedia" zu etablieren. S.644 in: Verbund Sozialwissenschaftliche Technikforschung, Mitteilungen 17. Frankfurt/M.

Luhmann, N., 1971: Öffentliche Meinung. S.9-33 in: Luhmann, N., Politische Planung. Opladen

Luhmann, N., 1981: Veränderungen im System gesellschaftlicher Kommunikation und die Massenmedien. S. 309-320 in: Luhmann, N., Soziologische Aufklärung 3. Opladen

Luhmann, N., 1990: Gesellschaftliche Komplexität und öffentliche Meinung. S. 170-182 in: Luhmann, N., Soziologische Aufklärung 5. Opladen

Luhmann, N., 1992: Die Beobachtung der Beobachter im politischen System: Zur Theorie offentlicher Meinung. S. 77-86 in: Wilke, J. (Hrsg.), Offentliche Meinung: Theorie, Methoden, Befunde. Beiträge zu Ehren von Elisabeth Noelle-Neumann. Freiburg

Luhmann, N., 1996: Die Realität der Massenmedien. Opladen

Marcinkowski, F, 1993: Publizistik als autopoietisches System. Politik und Massenmedien. Eine systemtheoretische Analyse. Opladen

Maresch, R., 1995: Mediatisierte Öffentlichkeiten. Leviathan 3: 394-416

Mast, C., 1986: Was leisten die Medien? Funktionaler Strukturwandel in den Kommunikationssystemen. Munchen

Matejovski, D., 1996: Von der Sinnstiftung zum Informationsdesign? - Die Kulturwissenschaften in den neuen Medienwelten. S. 252-272 in: Matejovski, D. /Kittler, F. (Hrsg.), Literatur im Informationszeitalter. Frankfurt/ M., New York 
McLuhan, M., 1968: Die Gutenberg-Galaxis. Düsseldorf Merten, K., 1977: Kommunikation. Eine Begriffs- und ProzeBanalyse. Opladen.

Merten, K., 1994: Evolution der Kommunikation. S.141162 in: Merten, K./Schmidt, S.J./Weischenberg, S. (Hrsg.), Die Wirklichkeit der Medien. Eine Einführung in die Kommunikationswissenschaft. Opladen

Merten K./Schmidt, S. J./Weischenberg, S. (Hrsg.), 1994: Die Wirklichkeit der Medien. Eine Einführung in die Kommunikationswissenschaft. Opladen

Meyrowitz, J., 1990: Überall und nirgends dabei. Die Fernsehgesellschaft. Weinheim, Basel

Müller, E., 1996: Massenhaft interaktiv? S. 146-159 in: Zacharias, W. (Hrsg.), Interaktiv. Im Labyrinth der Wirklichkeiten. Essen

Myers, D., 1987: „Anonymity is Part of the Magic“ Individual Manipulation of Computer-Mediated Communication Contexts. Qualitative Sociology 10: 251-266

Neidhardi, F., 1994: Öffentlichkeit, öffentliche Meinung, soziale Bewegungen. S. 7-41 in: Neidhardt, F. (Hrsg.), Öffentlichkeit, öffentliche Meinung, soziale Bewegungen. Sonderheft 34 der Kölner Zeitschrift für Soziologie und Sozialpsychologie. Opladen

Ong, W. J., 1977: Interfaces of the Word: Studies in the Evolution of Consciousness and Culture. Ithaca, New York

Peters, B., 1994: Der Sinn von Öffentlichkeit. S. 42-76 in: Neidhardt, F. (Hrsg.), Öffentlichkeit, öffentliche Meinung, soziale Bewegungen. Sonderheft 34 der Kölner Zeitschrift für Soziologie und Sozialpsychologie. Opladen

Prokop, D., 1972: Zum Problem von Produktion und Kommunikation im Bereich der Massenmedien. S.927 in: Prokop, D. (Hrsg.), Massenkommunikationsforschung 1. Frankfurt/M.

Prokop, D., 1973: Zum Problem von Konsumtion und Fetischcharakter im Bereich der Massenmedien. S. 9-44 in: Prokop, D. (Hrsg.), Massenkommunikationsforschung 2. Frankfurt/M.

Rafaeli, S., 1988: Interactivity. From New Media to Communication. S.110-134 in: Hawkins, R./Wiemann, J.M./Pingree, S. (Hrsg.), Advancing Communication Science. Merging Mass and Interpersonal Processes. Newbury Park

Renckstorf, K., 1989: Mediennutzung als soziales Handeln. Zur Entwicklung einer handlungstheoretischen Perspektive der empirischen (Massen-) Kommunikationsforschung. S.314-337 in: Kaase, M./Schulz, W. (Hrsg.), Massenkommunikation: Theorie, Methoden, Befunde, Sonderheft 30 der Kölner Zeitschrift für Soziologie und Sozialpsychologie. Opladen

Rheingold, H., 1995: Die Zukunft der Demokratie und die vier Prinzipien der Computerkommunikation. S. 189-197 in: Bollman, S. (Hrsg.), Kursbuch Neue Medien, Trend in Wirtschaft und Politik, Wissenschaft und Kultur. Mannheim

Riehm, U./Wingert, B., 1995: Multimedia. Mythen, Chancen und Herausforderungen. Karlsruhe

Rogers, E. M., 1986: Communication Technology. The New Media in Society. New York, London
Ronneberger, F., 1982: Neue Medien. Vorteile und Risiken für die Struktur der demokratischen Gesellschaft und den Zusammenhalt der sozialen Gruppen. Eine Literaturstudie, Schriften der Deutschen Gesellschaft für COMNET, Bd. 1. Konstanz

Rötzer, F., 1995: Telepolis. Urbanität im digitalen Zeitalter. Mannheim

Rötzer, F., 1995a: Konturen der ludischen Gesellschaft im Computerzeitalter. Vom Homo ludens zum ludo globi. S. 171-216 in: Rotzer, F. (Hrsg.), Schöne neue Welten? Auf dem Weg zu einer neuen Spielkultur. München

Rötzer, F. (Hrsg.), 1995b: Schöne neue Welten? Auf dem Weg zu einer neuen Spielkultur. München

Rötzer, F., 1996: Interaktion - das Ende herkömmlicher Massenmedien. S. 119-134 in: Maresch, R. (Hrsg.), Medien und Öffentlichkeit. Positionierungen Symptome Simulationsbrüche. München

Schenk, M., 1991: Massenkommunikation und Interpersonale Kommunikation. S. 405 -417 in: Kaase, M./Schulz, W. (Hrsg.), Massenkommunikation. Theorie, Methoden, Befunde. Opladen.

Schmidt, S.J., 1994: Kognitive Autonomie und soziale Orientierung. Frankfurt/M.

Schmidt, S.J., 1994a: Die Wirklichkeit des Beobachters. S. 212-236 in: Merten, K./Schmidt, S.J./Weischenberg, S. (Hrsg.), Die Wirklichkeit der Medien. Eine Einfuhhrung in die Kommunikationswissenschaft. Opladen

Schmidt, S.J., 1995: Cyber als Oikos? Oder: ernste Spiele. S. 69-90 in: Rötzer, F. (Hrsg.), Schöne neue Welten? Auf dem Weg zu einer neuen Spielkultur. München

Schulze, G., 1995: Das Medienspiel. S. 363-379 in: MüllerDoohm,S./Neumann-Braun, K. (Hrsg.), Kulturinszenierungen. Frankfurt/M.

Seeger, P., 1995: Strukturveränderungen des Rundfunks in Europa durch technischen Wandel. S. 103-132 in: Hoffmann-Riem, W./Vesting, T. (Hrsg.), Perspektiven der Informationsgesellschaft. Baden-Baden

Spangenberg, P.M., 1988: TV, Hören und Sehen. S. 776789 in: Gumbrecht, H.U./Pfeiffer, K.L. (Hrsg.), Materialităt der Kommunikation. Frankfurt/M.

Spangenberg, P.M., 1991: Mediale Kopplungen und die Konstruktivităt des Bewußtseins. S. 791-808 in: Gumbrecht, H.U./Pfeiffer, K.L. (Hrsg.), Paradoxien, Dissonanzen, Zusammenbrüche. Frankfurt/M.

Spangenberg, P.M., 1996: Komplexitătsebenen moderner Öffentlichkeit. Uber die mediale Emergenz kommunikativer Wirklichkeitskonstruktion und ihre Verfremdung durch technische Visualisierung. S. 263-277 in: Maresch, R. (Hrsg.), Medien und Öffentlichkeit. Positionierungen Symptome Simulationsbríche. München Vogelsang, W., 1996: Abenteursuche im virtuellen Raum. S. 232-247 in: Zacharias, W. (Hrsg.), Interaktiv. Im Labyrinth der Wirklichkeiten. Essen

Vorderer, P., 1995: Will das Publikum neue Medien(angebote)? Medienpsychologische Thesen über die Motivation zur Nutzung neuer Medien. Rundfunk und Fernsehen 43: 494-505

Wagner, I., 1993: Neue Reflexivităt. Technisch vermittelte Handlungsrealităten in Organisationen. S.7-66 in: Wagner, I. (Hrsg.), Kooperative Medien. Informations- 
technische Gestaltung moderner Organisationen. Frankfurt/M., New York

Wehner, J., 1996: Vom Zuschauer zum Mitspieler - Ende der Massenkultur? (unveröff. Diss.)

Wehner, J., 1997: Medien als Kommunikationspartner Zur Entstehung elektronischer Schriftlichkeit im Internet. S. 12-29 in: Gräf, L./Krajewski, M. (Hrsg.), Zur Soziologie des Internet. Frankfurt/M.

Weingarten, R., 1996: Sprachnormen, Verrechtlichung und die Mediatisierung von Kommunikation. S. 15-37 in: Matejovski, D./Kittler, F. (Hrsg.), Literatur im Informationszeitalter. Frankfurt/M.

Welsch, W., 1995: Künstliche Paradiese? Betrachtungen zur Welt der elektronischen Medien und anderen Welten. S. 71-95 in: Baake, D./Röll, F.J. (Hrsg.), Weltbilder Wahrnehmung Wirklichkeit - der àsthetisch organisierte Lernprozeß. Opladen
Wetzstein, T.A./Dahm, H./Steinmetz, L./Eckert, R., 1995: Datenreisende. Die Kultur der Computernetze. Opladen

Wetzel, K., 1995: The TV-Culture of Tomorrow: Vom Zuschauer zum User. S. 18-25 in: Rötzer, F. (Hrsg.), Schöne neue Welten? Auf dem Weg zu einer neuen Spielkultur. München

Williams, F.Pavlic, J.V. (Hrsg.), 1994: The People's right to know: media, democracy, and the information highway. Hillsdale, New Jersey

Wingert, B., 1996: Kann man Hypertexte lesen? S. 185219 in: Matejovski, D./Kittler, F. (Hrsg.), Literatur im Informationszeitalter. Frankfurt/M., New York

Zacharias, W., (Hrsg.), 1996: Interaktiv. Im Labyrinth der Wirklichkeiten. Essen 